\title{
Parasympathetic response in chick myocytes and mouse heart is controlled by SREBP
}

\author{
Ho-Jin Park, ${ }^{1}$ Serban P. Georgescu, ${ }^{1}$ Chuang Du, ${ }^{2}$ Christopher Madias, ${ }^{1,3}$ \\ Mark J. Aronovitz, ${ }^{1}$ C. Michael Welzig, ${ }^{4}$ Bo Wang, ${ }^{5}$ Ulrike Begley, ${ }^{1}$ Yali Zhang, ${ }^{1}$ \\ Robert O. Blaustein, ${ }^{1,3}$ Richard D. Patten,1,3 Richard H. Karas, ${ }^{1,3}$ Herbert H. Van Tol, 6 \\ Timothy F. Osborne,7 Hitoshi Shimano, ${ }^{8}$ Ronglih Liao, ${ }^{5}$ Mark S. Link, ${ }^{3}$ and Jonas B. Galper ${ }^{1,3}$ \\ ${ }^{1}$ Molecular Cardiology Research Institute, Department of Medicine, Tufts-New England Medical Center and Tufts University School of Medicine, \\ Boston, Massachusetts, USA. ${ }^{2}$ Center for Neuroscience Research, Department of Neuroscience, Tufts University School of Medicine, \\ Boston, Massachusetts, USA. ${ }^{3}$ Cardiology Division, Department of Medicine, Tufts-New England Medical Center, Boston, Massachusetts, USA. \\ ${ }^{4}$ Children's Heart Program, Medical University of South Carolina, Charleston, South Carolina, USA. ${ }^{5}$ Department of Medicine, Brigham and Woman's Hospital, \\ Boston, Massachusetts, USA. ${ }^{\circ}$ Department of Psychiatry, University of Toronto, Toronto, Ontario, Canada. ${ }^{7}$ Department of Molecular Biology and Biochemistry, \\ School of Biological Sciences, UC Irvine, Irvine, California, USA. ${ }^{8}$ Department of Internal Medicine, University of Tsukuba, Tsukuba, Japan.
}

\begin{abstract}
Parasympathetic stimulation of the heart, which provides protection from arrhythmias and sudden death, involves activation of the $\mathrm{G}$ protein-coupled inward rectifying $\mathrm{K}^{+}$channel GIRK1/4 and results in an acetylcholine-sensitive $\mathrm{K}^{+}$current, $\mathrm{I}_{\mathrm{KACh}}$. We describe a unique relationship between lipid homeostasis, the lipid-sensitive transcription factor SREBP-1, regulation of the cardiac parasympathetic response, and the development of ventricular arrhythmia. In embryonic chick atrial myocytes, lipid lowering by culture in lipoprotein-depleted serum increased SREBP-1 levels, GIRK1 expression, and $\mathrm{I}_{\mathrm{KACh}}$ activation. Regulation of the GIRK1 promoter by SREBP-1 and lipid lowering was dependent on interaction with 2 tandem sterol response elements and an upstream E-box motif. Expression of dominant negative SREBP-1 (DN-SREBP-1) reversed the effect of lipid lowering on $\mathrm{I}_{\mathrm{KACh}}$ and GIRK1. In SREBP-1 knockout mice, both the response of the heart to parasympathetic stimulation and the expression of GIRK1 were reduced compared with WT. $\mathrm{I}_{\mathrm{KACh}}$, attenuated in atrial myocytes from SREBP-1 knockout mice, was stimulated by SREBP-1 expression. Following myocardial infarction, SREBP-1 knockout mice were twice as likely as WT mice to develop ventricular tachycardia in response to programmed ventricular stimulation. These results demonstrate a relationship between lipid metabolism and parasympathetic response that may play a role in arrhythmogenesis.
\end{abstract}

\section{Introduction}

The balance between the response of the heart to sympathetic and parasympathetic stimuli not only determines the rate and force of contraction but also plays a role in regulating cardiac excitability and the genesis of cardiac arrhythmias. Parasympathetic stimulation of the heart has been shown to be protective against the development of life-threatening arrhythmias in animal models for myocardial infarction (MI) (1). Following MI, stimulation of the vagus nerve protected the canine heart from pacing-induced ventricular flutter. Furthermore, analysis of heart rate variability has demonstrated a relationship between sudden death and diurnal variation of parasympathetic regulation of the heart (2), while parasympathetic dysfunction in patients with diabetic autonomic neuropathy (DAN) has been associated with the development of arrhythmias and sudden death $(2,3)$.

Parasympathetic regulation of heart rate involves binding of acetylcholine to $\mathrm{M}_{2}$-muscarinic receptors localized primarily on atrial myocytes, which stimulates the dissociation of the heterotrimeric $\mathrm{G}$ protein $\mathrm{G}_{\mathrm{i} 2}$ into $\mathrm{G} \alpha_{\mathrm{i} 2}$ and $\mathrm{G} \beta \gamma$ subunits. The dissociated $\mathrm{G} \beta \gamma$

Nonstandard abbreviations used: DAN, diabetic autonomic neuropathy; DN-SREBP-1, dominant negative SREBP-1; EDD, end-diastolic diameter; ESD, end-systolic diameter; GIRK, G protein-coupled inward rectifying $\mathrm{K}^{+}$channel; $\mathrm{I}_{\mathrm{KACh}}$, acetylcholine-sensitive $\mathrm{K}^{+}$current; I-V, current-voltage; LPDS, lipoprotein-depleted serum; MI, myocardial infarction; SCAP, SREBP cleavage-activating protein; SRE, sterol regulatory element; VERP, ventricular effective refractory period; VF, ventricular fibrillation; VT, ventricular tachycardia.

Conflict of interest: R.O. Blaustein receives consulting income from CV Therapeutics. Citation for this article: J. Clin. Invest. 118:259-271 (2008). doi:10.1172/JCI32011. subunit activates the $\mathrm{G}$ protein-coupled inward rectifying $\mathrm{K}^{+}$channel (GIRK1) $2 /(\mathrm{GIRK} 4)_{2}$, resulting in an acetylcholine-stimulated $\mathrm{K}^{+}$current, $\mathrm{I}_{\mathrm{KACh}}$. Stimulation of $\mathrm{I}_{\mathrm{KACh}}$ hyperpolarizes the myocyte membrane, increasing the duration of diastolic depolarization and resulting in a decrease in heart rate $(4,5)$.

Lipid lowering by HMG-CoA reductase inhibitors has become a major therapeutic end point in the treatment and prevention of atherosclerotic cardiovascular disease. In an in vitro model for lipid lowering in which embryonic chick atrial myocytes were cultured in lipoprotein-depleted serum (LPDS), lipid lowering resulted in a marked increase in the response of beat rate to muscarinic stimulation and a reciprocal decrease in the response of beating to $\beta$-adrenergic stimulation (6). These changes were associated with an increase in the expression of the $\mathrm{M}_{2}$-muscarinic receptor, $\mathrm{Ga}_{\mathrm{i} 2}$ and GIRK1 (7). These findings suggested the hypothesis that a unique relationship might exist between lipid metabolism and the parasympathetic response of the heart.

SREBPs are a family of 3 transcription factors, basic helix-loophelix leucine zipper-containing proteins, that regulate expression of genes involved in lipid homeostasis and glucose metabolism. SREBPs are synthesized in the ER as 130-kDa precursor molecules that are transported to the Golgi by a sterol-sensitive SREBP cleavage-activating protein (SCAP) $(8,9)$. In the Golgi, the precursor is processed via a 2 -step sequential proteolytic cleavage to produce a 480-amino acid transcription factor, which is transported to the nucleus. The expression, transport and processing of these proteins are subject to feedback regulation by sterols. In the presence of cholesterol, SCAP and the SREBP precursors remain anchored 
A

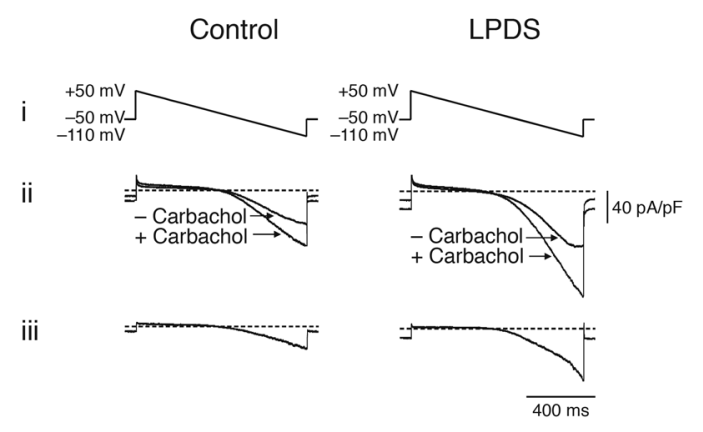

B

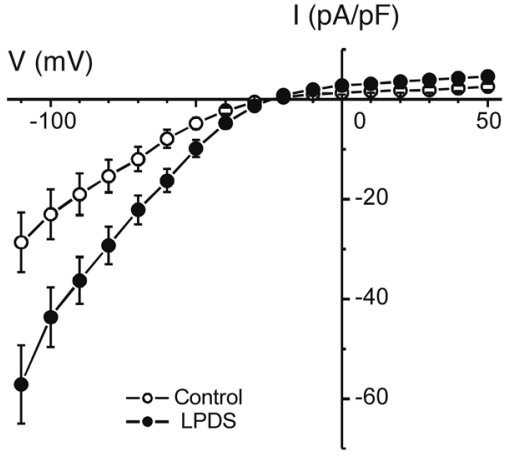

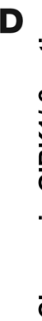

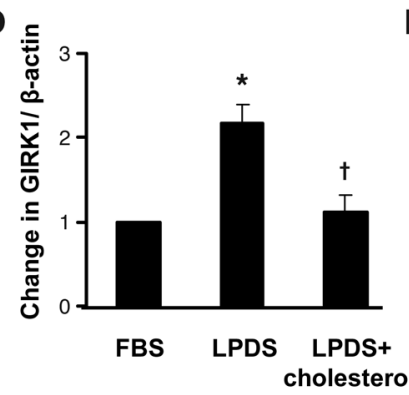

E

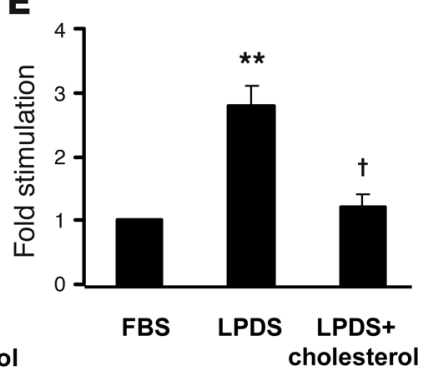

Figure 1

Effect of lipid lowering on $I_{\text {KACh }}$ and the expression of GIRK1 in cultured embryonic chick atrial myocytes. (A) I-V relationship of the carbachol-induced whole-cell currents elicited from a 1-second voltage ramp with a voltage continuously changing from +50 to $-110 \mathrm{mV}$, from a holding potential of $-50 \mathrm{mV}$ (i). Current in a typical atrial myocyte in a high extracellular $\mathrm{K}^{+}(50 \mathrm{mM}), 0 \mathrm{Ca}^{+2}$ bath with and without 20 $\mu \mathrm{M}$ carbachol (ii). Current generated by subtracting the trace obtained prior to and after the addition of carbachol (iii). (B) An I-V plot constructed from a series of data points obtained from the carbachol current responses at given voltages. Average carbachol-induced current densities measured in myocytes cultured in FBS or LPDS. Each point is the mean of measurements from 3 independent cultures. (C) Western blot analysis of GIRK1 expression in atrial myocytes cultured in FBS, LPDS, or LPDS supplemented with a mixture of $10 \mu \mathrm{M}$ cholesterol and $1 \mu \mathrm{M}$ 25-hydroxycholesterol. (D) Fold stimulation of GIRK1 protein determined by densitometry scanning of 4 independent experiments similar to that in C. Data represent the relative intensity of GIRK1 normalized to $\beta$-actin with the value in FBS taken as 1. (E) Fold stimulation of GIRK1 promoter activity above levels in FBS. Data are normalized to $\beta$-gal activity and are the mean of 4 independent determinations. ${ }^{\star} P<0.05$, ${ }^{\star *} P<0.01$ compared with control; ${ }^{\dagger} P<0.05$ compared with LPDS.

to ER membrane proteins, Insigs (10). In lipoprotein-depleted cells, SCAP is released from Insigs and transports the SREBP precursor to the Golgi, facilitating its proteolysis. In the nucleus, SREBPs may form a transcription complex by binding to an E-box, a palindromic motif, the consensus recognition element for basic helix-loop-helix leucine zipper transcription factors. Alternatively, SREBPs bind to a sterol regulatory element (SRE), which may occur as multiple tandem SREs often associated with an E-box motif, as described for the acetyl-CoA synthase promoter (11). Generic coactivators such as Sp1 and YY-1 have been shown to be required for optimal activity of the LDL receptor and the HMG-CoA synthase promoters, respectively (12). We have previously presented data supporting the conclusion that SREBP-1 might regulate the expression of $\mathrm{G \alpha}_{\mathrm{i} 2}$ in cultured atrial myocytes in response to lipid lowering (13). However, little is known about the regulation of gene expression by sterols and SREBPs in the heart.

Here, we describe what we believe is a novel relationship between lipid metabolism and the response of the heart to parasympathetic stimulation and report a new role for SREBP in the regulation ously demonstrated an increase in parasympathetic responsiveness in atrial myocytes grown in LPDS (6). To determine whether this effect was due to an increase in $\mathrm{I}_{\mathrm{KACh}}$, membrane currents from atrial myocytes cultured in FBS and LPDS were compared (Figure 1A). The current-voltage (I-V) relationships demonstrated inward rectification with a reversal potential at $-28 \mathrm{mV}$ consistent with a $\mathrm{K}^{+}$dependent current at $50 \mathrm{mM}$ extracellular $\mathrm{K}^{+}$. Lipoprotein depletion increased the peak inward current from $-23 \pm 4 \mathrm{pA} / \mathrm{pF}(n=7)$ in cells cultured in FBS to $-62 \pm 8 \mathrm{pA} / \mathrm{pF}(n=6 ; P<0.01)$ in cells cultured in LPDS with no change in reversal potential (Figure 1B). Lipid lowering increases the expression of GIRK1 in atrial myocytes. In order to determine whether the increase in $\mathrm{I}_{\mathrm{KACh}}$ in response to lipid lowering is due to an increase in expression of GIRK1, embryonic chick atrial myocytes were cultured in the presence of either FBS, LPDS, or LPDS plus cholesterol, and GIRK1 expression was determined by Western blot analysis. Growth in LPDS resulted in a $2.2 \pm 0.3$-fold $(n=4 ; P<0.05)$ increase in expression of GIRK1 that was reversed by the addition of cholesterol to the culture medium (Figure 1, C and D). To determine whether lipid lowering regu- 
A

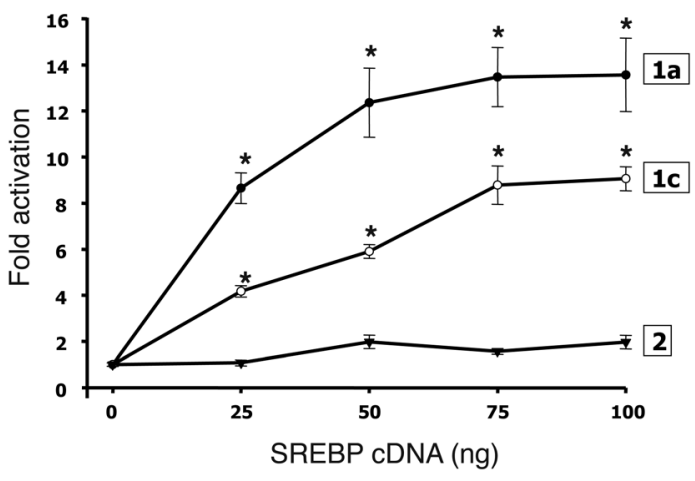

B

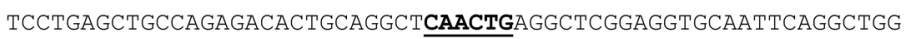
E Box

CTGGTGGAGCAGCGGGGGAAGGCAGGGACAGGCGGGGCTAGCGCGGCTCTGCAGCTGATC Sp1 E Box

ACACGCACCACCACACACACCACATTCCTGCTGCCCCGGCTTGCCCCTCGCATCTTCCAC

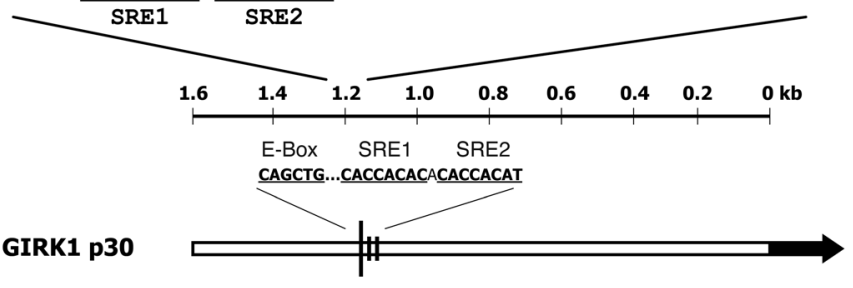

GIRK1 p30XH

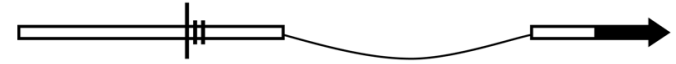

GIRK1 p30FX

GIRK1 p21

GIRK1 p21XH

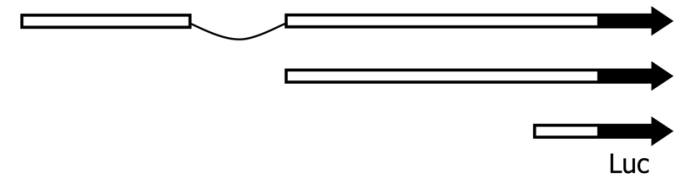

C

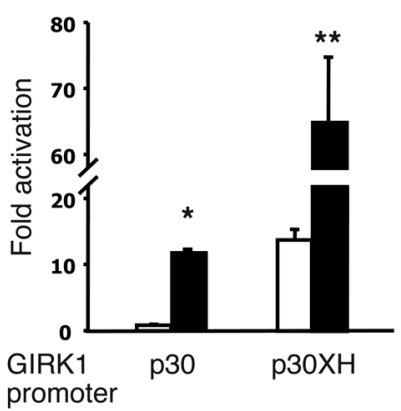

D

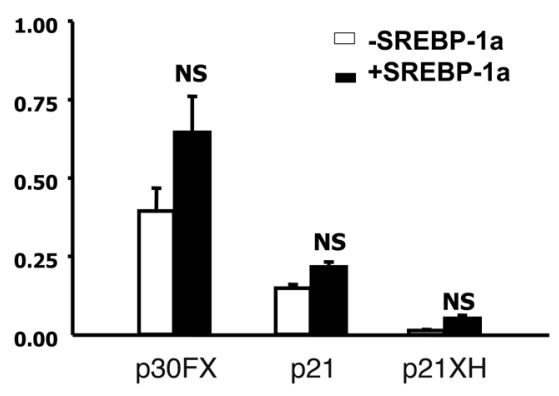

E

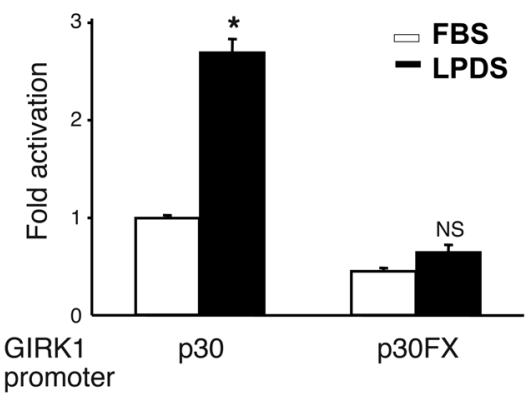

Figure 2

Cis-acting elements required for SREBP regulation of the GIRK1 promoter. (A) Specificity of SREBP stimulation of GIRK1 promoter activity. Atrial myocytes were cotransfected with a construct containing $1.6 \mathrm{~kb}$ of the GIRK1 promoter reporter and increasing amounts of a construct expressing either SREBP-1a, -1c, or -2. (B) Upper panel: Nucleotide sequence of the GIRK1 promoter from -1070 to -1209. Lower panel: Deletion constructs of the GIRK1 promoter. (C and D) Effect of deletion mutants of the GIRK1 promoter on SREBP-1a stimulation of promoter activity. Data are plotted as fold stimulation above basal level of the p30 promoter in the absence of SREBP-1a, taken as 1. Data represent the mean of 4 determinations carried out in triplicate. Note that scales of the $y$ axes in $\mathbf{C}$ and $\mathbf{D}$ are different. (E) Effect of FX deletion in the GIRK1 promoter on LPDS stimulation of promoter activity. Cells cultured in FBS or LPDS were transfected with either the full-length GIRK1 promoter (p30) or the FX deletion mutant. The activity of the p30 promoter in FBS was considered as $1 .{ }^{*} P<0.05,{ }^{* *} P<0.001$ compared with control.

lates GIRK1 expression at the level of transcription, we analyzed the activity of a GIRK1 promoter-luciferase reporter transfected into atrial myocytes cultured in FBS, LPDS, or LPDS plus cholesterol. LPDS induced a $2.8 \pm 0.4$-fold $(n=6 ; P<0.01)$ increase in GIRK1 promoter activity compared with FBS (Figure 1E). Incubation with cholesterol essentially reversed the effect of lipid lowering on GIRK1 promoter activity. Given that cholesterol lowering has been shown to stimulate sterol responsive pathways and that this stimulation is reversed by the addition of cholesterol to the medium (16), these data suggested that expression of GIRK1 might be under the control of sterols.

SREBP-1 regulates the expression of GIRK1 at the level of transcription. We had previously demonstrated that growth of embryonic chick atrial myocytes in LPDS increased the level of SREBP-1 in the nucleus (13). To determine whether the increase in GIRK1 levels in cells cultured in LPDS was due to SREBP regulation of GIRK1 expression, atrial myocytes were cotransfected with the
GIRK1 promoter-luciferase reporter and increasing concentrations of cDNAs coding for either SREBP-2, -1a, or -1c. SREBP-1a and $-1 \mathrm{c}$ stimulated GIRK1 promoter activity $13.2 \pm 1.3$-fold and $9.2 \pm 1.1$-fold, respectively $(n=4 ; P<0.005)$, while SREBP-2 had no significant effect (Figure 2A). This finding was consistent with functional differences between SREBP-2 and SREBP-1a and -1c: SREBP-1a and - $1 \mathrm{c}$ are more specific for the regulation of fatty acid and glucose metabolism while SREBP-2 is more specific for the control of cholesterol homeostasis (17).

SREBP-1 regulation of GIRK1 promoter activity requires 2 tandem SREs and an upstream E-box. Analysis of the GIRK1 promoter demonstrated the presence of 2 tandem 8-bp regions at position -1246 to -1230 separated by a single adenosine with the sequences CACCA$\mathrm{CAC}$ and CACCACAT. Both of these sequences are highly homologous to the consensus sequence for SRE, CACCSYAC, where $\mathrm{S}$ is either a $\mathrm{G}$ or $\mathrm{C}$ and $\mathrm{Y}$ is a pyrimidine. These putative SREs were 11 bps downstream from an E-box with the sequence CAGCTG 
A

wild type

point mutation 1

point mutation 2

point mutation 1,2

E-box / wild type

E-box / point mutation 1,2
E-box

(wt)

$\overline{\text { CAGCTG}}$ ATCACACGCACCACCACACA $\overline{\text { CACCACAT }}$

(S1) CAGCTGATCACACGCACAACCACACACACCACAT

(S2) CAGCTGATCACACGCACCACCACACAAACCACAT

$(S 1,2)$

CAGCTGATCACACGCACAACCACACAAACCACAT

(E)

TGTAACATCACACGCACCACCACACACACCACAT

(ES1,2)

TGTAACATCACACGCACAACCACACAAACCACAT

B
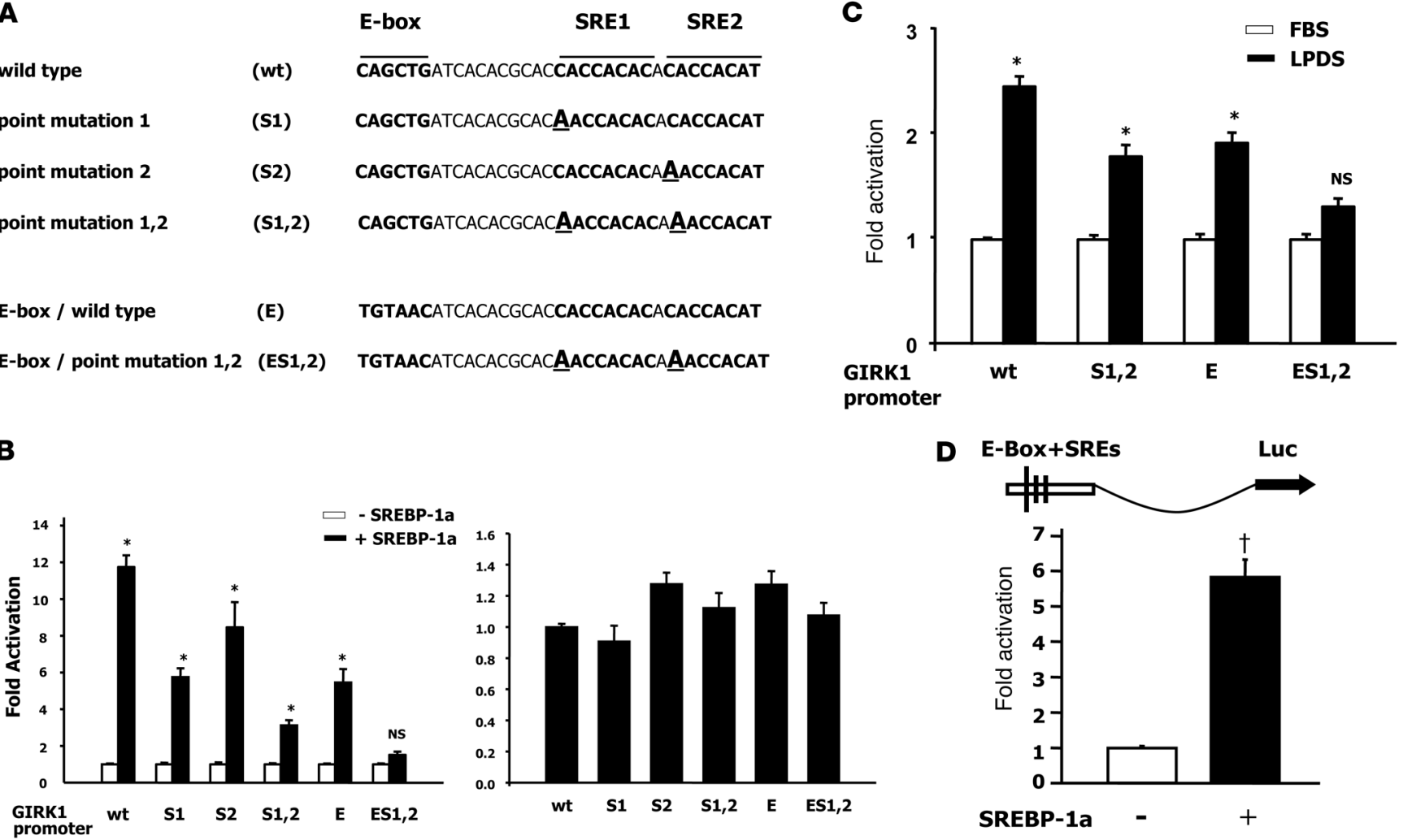

E

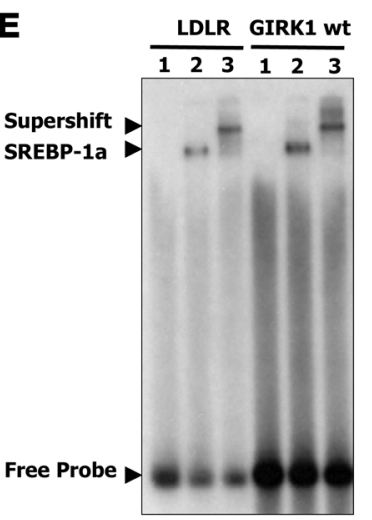

$\frac{\text { LDLR }}{123} \frac{\text { GIRK1 wt }}{123}$

F

F $\frac{W T}{123} \frac{S 1}{123} \frac{S 2}{123} \frac{S 1,2}{123}$

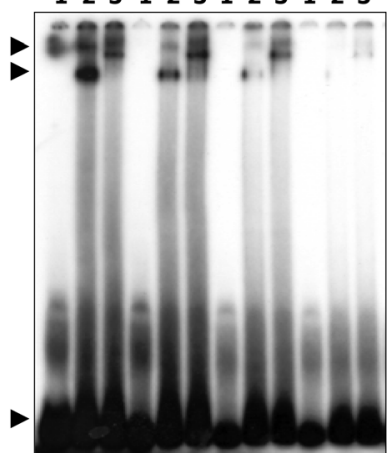

G

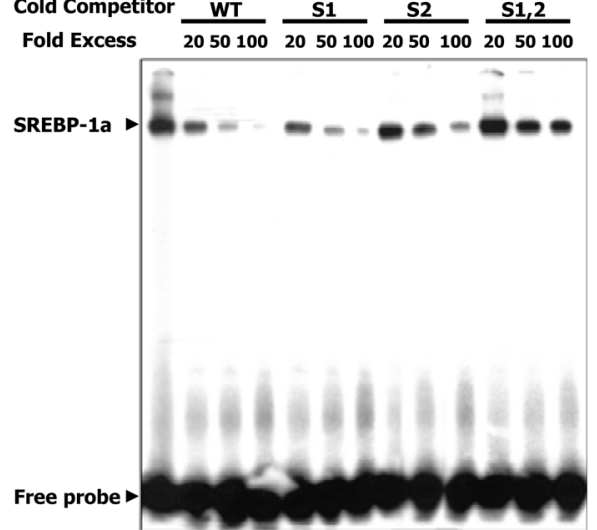

H

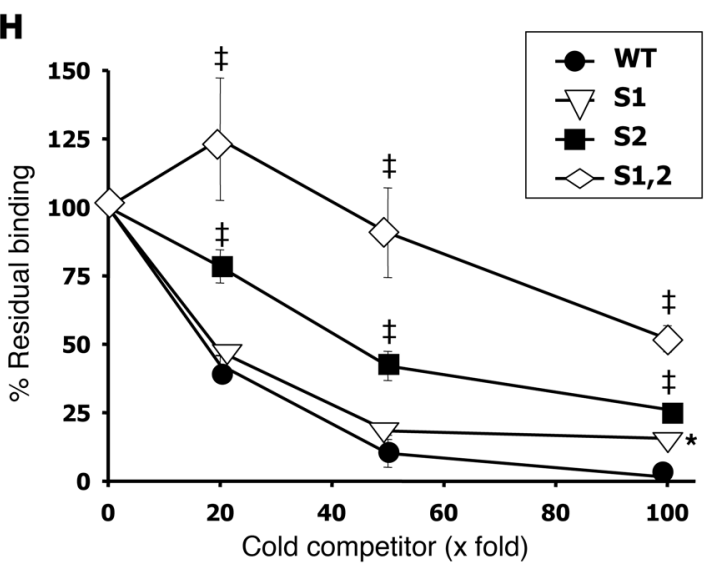

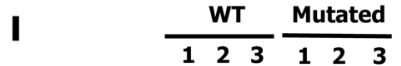

Supershift SREBP-1a

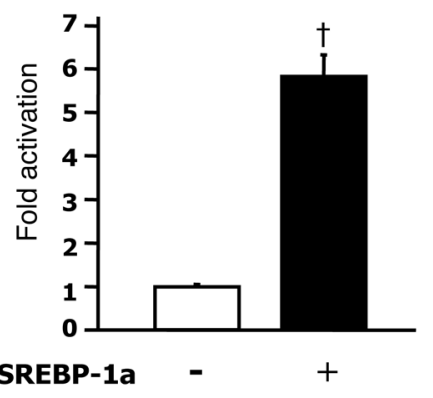

SREBP-1a - + 


\section{Figure 3}

Functional significance of 2 tandem SREs and upstream E-box in the GIRK1 promoter. (A) Constructs containing point mutations in SRE-1 (S1), SRE-2 (S2), or SRE-1 and -2 (S1,2); replacement of the E-box with a scrambled sequence $(E)$ or point mutations in SRE-1, SRE-2, and the mutant E-box $(E S 1,2)$ were ligated to a luciferase reporter. Bold underlined letters indicate mutated bases. (B) Left panel: Effect of point mutations in putative SREs and E-box on SREBP-1a-stimulated GIRK1 promoter activity $(n=4 ; P<0.01)$. Right panel: Basal activities of the constructs containing point mutations. $n \geq 5$. (C) Effect of point mutations on LPDS stimulation of GIRK1 promoter activity. Data represent the mean of 7 experiments carried out in triplicate; * $P<0.05$. (D) SREBP-1a stimulates a construct containing both SREs and the upstream E-box of the GIRK1 promoter. $n=7{ }^{\dagger} P<0.001$. (E) EMSA demonstrating binding of SREBP-1a to SRE-1 and SRE-2 in the GIRK1 promoter as described in Methods. Oligonucleotides containing the SRE site of the LDL receptor promoter were used as a positive control; 1, oligo alone; 2, oligo plus SREBP-1a; 3 , oligo plus SREBP-1a plus anti-SREBP-1 antibody. (F) As in D, except binding was to oligos containing either WT or mutant SREs from the GIRK1 promoter. (G) Competition assay for binding of oligos containing point mutations in SRE-1 and SRE-2. Fold excess of the unlabeled competitor is indicated. (H) Quantification of data in G. Data are plotted as the ratio of residual binding to binding in the absence of competitor. $n=4$; ${ }^{\ddagger} P<0.01$. (I) Binding of SREBP-1a to the E-box in the GIRK1 promoter with oligonucleotides containing WT and mutant E-boxes.

and adjacent to an Sp1 site 14 bps upstream from the E-box (Figure $2 \mathrm{~B}$ ). In order to determine whether these SREs were functionally significant, we made a series of deletion mutants of the GIRK1 promoter and determined the ability of these constructs to respond to overexpression of SREBP-1a (Figure 2, B-D). Deletion of the region of the promoter downstream from the putative SREs, p30XH, increased basal promoter activity $13.9 \pm 1.6$-fold $( \pm$ SEM,$n=4)$ above the basal activity of the full-length p30 promoter, consistent with the presence of a repressor binding site in the $\mathrm{XH}$ region (Figure $2 \mathrm{C}$ ). While SREBP-1a stimulated the activity of the full-length GIRK1 p30 promoter $11.9 \pm 1.0$-fold $(P<0.05)$, SREBP-1 stimulated the $\mathrm{p} 30 \mathrm{XH}$ promoter $4.7 \pm 0.7$-fold $(n=4)$ above its own basal and $65.0 \pm 9$-fold $(P<0.001)$ above the basal activity of the full-length promoter. Deletion of a region of the GIRK1 promoter, $\mathrm{p} 30 \mathrm{FX}$, which included the 2 tandem putative SREs and the downstream half of the proximal E-box, abolished SREBP-1a stimulation of GIRK1 promoter activity while decreasing basal promoter activity by $0.4 \pm 0.07$-fold (Figure $2 \mathrm{C}$ ). Finally, the increase in GIRK1 promoter activity in response to LPDS was also abolished by the p30FX deletion (Figure 2D).

Furthermore, a single point mutation in SRE-1 (S1) decreased SREBP-1a-stimulated GIRK1 promoter activity by $56.2 \% \pm 4.4 \%$, while a single point mutation in SRE-2 (S2) diminished the SREBP-1astimulated promoter activity by $31.3 \% \pm 12.6 \%$. Point mutations in both SRE-1 and SRE-2 (S1,2) decreased SREBP-1a stimulation of GIRK 1 promoter activity by $79.4 \% \pm 3.1 \%$, suggesting that the contribution of each putative SRE might be additive (Figure 3, A and B). To determine the role of the upstream E-box on SREBP-1 regulation of GIRK1 promoter activity, the E-box sequence was mutated (Figure $3 \mathrm{~A}$ ), resulting in a $59.1 \% \pm 6.8 \%$ decrease in promoter activity (Figure $3 \mathrm{~B}$ ). Combined point mutations in SRE-1, SRE-2, and the E-box $(E S 1,2)$ decreased the SREBP-1a effect on GIRK1 promoter activity to control levels. None of these mutations had a significant effect on basal promoter activity (Figure 3B). These data suggest that SREBP-1a regulation of the GIRK1 promoter involves the interaction of both the 2 tandem SREs and the upstream E-box.

Although neither S1 nor S2 alone had an effect on LPDS stimulation of GIRK1 promoter activity (not shown), the S1,2 mutation decreased LPDS activation of GIRK1 promoter activity by $46.6 \% \pm 7.5 \%$ (Figure 3C). Mutation of the E-box decreased LPDS stimulation of GIRK1 promoter activity by $37.8 \% \pm 6.7 \%$. Combined mutations in both SREs and the E-box decreased LPDS stimulation by $79 \% \pm 5.3 \%$, which was not significantly different from basal promoter activity in the presence of FBS (Figure 3C). Finally, we performed reporter assays using a construct (E-SRE-Luc) that includes both SREs and the upstream E-box of the GIRK1 promoter ligated to pGL2, which contains the minimal thymidine kinase promoter. As expected, E-SRE-Luc showed a strong induction by SREBP-1a, confirming the importance of this region in the regulation of GIRK1 by SREBP (Figure 3D).

SREBP-1 binding to the GIRK1 promoter. To determine whether the effect of these point mutations in the regulation of the GIRK1 promoter reflected actual changes in the binding of SREBP-1a, we performed EMSAs. Data summarized in Figure 3E demonstrated that purified SREBP-1a formed a complex with the WT probe containing the 2 tandem SREs and that incubation with an antibody specific for SREBP-1 supershifted the complex. Thus, there was no binding in the absence of purified SREBP-1, a shift in the migration of the probe in the presence of purified SREBP-1 and a supershift when antibody to SREBP-1 was incubated with the probe and the purified SREBP-1. At the same time, oligonucleotides containing individual point mutations in either SRE-1 (S1) or SRE-2 (S2) demonstrated decreased complex formation with purified SREBP-1a, while SREBP-1a binding to an oligonucleotide containing point mutations in both SRE-1 and SRE-2 (S1,2) was practically abolished (Figure 3F). Finally, in order to further determine the specificity of the interaction between SREBP-1a and the tandem SREs in the GIRK1 promoter, we performed competition experiments. Data in Figure 3, G and H, demonstrated that S1 and S2 competed in a dosedependent manner with binding of the WT probe, while S1,2 had little effect on complex formation with significant residual binding (52\%), even in the presence of 100 -fold excess of the competitor. Taken together, these results demonstrate that SREBP-1 binds specifically to the GIRK1 promoter and regulates its activity.

To determine whether the effects of SREBP-1 on GIRK1 promoter also involved binding to the upstream E-box, EMSAs for the binding of purified SREBP-1a to oligonucleotides containing either the WT sequence or the mutated E-box hexamer were compared. Purified SREBP-1a formed a complex with the WT E-box probe, which was supershifted by an antibody specific for SREBP-1. In contrast, binding to an oligonucleotide containing the mutated E-box was markedly decreased (Figure 3I).

SREBP-1 regulates the expression of GIRK1 in response to lipid lowering. To directly determine the role of SREBP in the regulation of GIRK1 expression, atrial myocytes cultured in LPDS were cotransfected with the GIRK1-luciferase reporter and a construct expressing dominant negative SREBP-1 (DN-SREBP-1). Expression of DN-SREBP-1 completely reversed LPDS stimulation of GIRK1 promoter activity (Figure 4A). Furthermore, in atrial myocytes infected with adenovirus expressing a control virus (Ad- $\beta$-gal), growth in LPDS stimulated GIRK1 protein levels $2.2 \pm 0.28$-fold ( \pm SEM; 
A

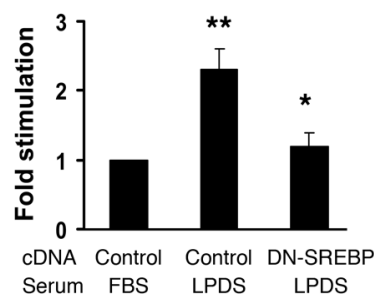

\section{B}

C

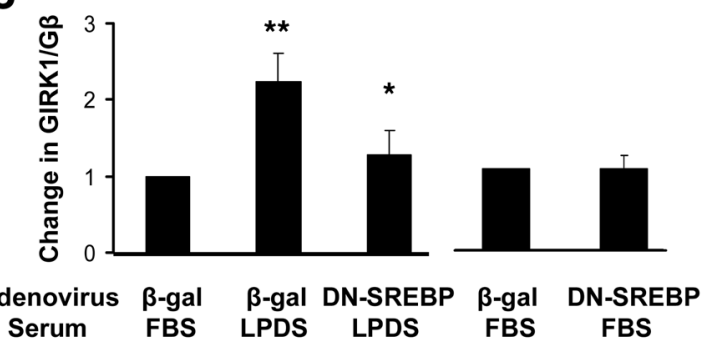

D

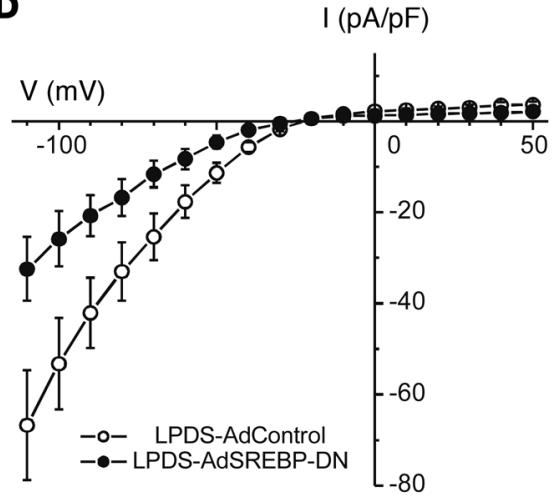

$P<0.01)$ above that in cells cultured in FBS. However, adenoviral expression of DN-SREBP-1 completely reversed the effect of LPDS on GIRK1 protein expression (Figure 4, B and C) while having no significant effect on GIRK1 expression in cells cultured in FBS (Figure 4, B and C). Finally, adenoviral expression of SREBP-1 in atrial myocytes grown in FBS increased GIRK1 protein expression to levels similar to those in cells cultured in LPDS (data not shown).

Overexpression of DN-SREBP-1 in atrial myocytes reverses LPDS stimulation of $I_{K A C b}$. Data summarized in Figure 1 demonstrated that culture of atrial myocytes in LPDS resulted in an increase in both $\mathrm{I}_{\mathrm{KACh}}$ and the expression of GIRK1 (Figure 1, B and D). We had previously demonstrated that growth of atrial myocytes in LPDS increased the levels of SREBP in the nucleus (13). To determine the role of SREBP in the regulation of $\mathrm{I}_{\mathrm{KACh}}$ by LPDS, atrial myocytes cultured in LPDS were infected with adenovirus expressing either $\beta$-gal or DN-SREBP-1. $\mathrm{I}_{\mathrm{KACh}}$ in cells expressing $\beta$-gal cultured in LPDS was $-66.7 \pm 12 \mathrm{pA} / \mathrm{pF}( \pm \mathrm{SEM}, n=7)$, which was not significantly different from $\mathrm{I}_{\mathrm{KACh}}$ in uninfected atrial myocytes in cells cultured in

\section{Figure 4}

DN-SREBP-1 blocks the effect of LPDS on GIRK1 expression and I IACh. (A) DN-SREBP-1 reverses the LPDS-mediated increase in GIRK1 promoter activity. Reporter assays were carried out in atrial myocytes cultured in FBS or LPDS and transfected with GIRK1-Luc plus a plasmid expressing either $\beta$-gal or SREBP-1c. Data are the mean of 4 experiments carried out in triplicate. ${ }^{*} P<0.01$ compared with FBS; ${ }^{\star} P<0.05$ compared with LPDS. (B) DN-SREBP-1 blocks the LPDSmediated increase in GIRK1 protein levels, while having no effect on GIRK1 expression in cells cultured in FBS. Embryonic chick atrial myocytes were infected with an adenoviral vector expressing GFP plus DN-SREBP-1 or GFP plus $\beta$-gal at the time of plating (MOI of 20) as described in Methods. On the third culture day, cells were harvested and expression of GIRK1 determined by Western blot analysis. Fluorescence microscopy demonstrated that $80 \%-90 \%$ of cells were GFP positive. (C) Densitometry scanning of 4 experiments similar to that in B. Data are normalized to the expression of $\mathrm{G} \beta .{ }^{*} P<0.01$ compared with FBS; ${ }^{*} P<0.05$ compared with LPDS. (D) Effect of DN-SREBP-1 on $I_{K A C h}$ in cells cultured in LPDS. Embryonic atrial myocytes were cultured in LPDS cells infected with adenovirus expressing GFP plus DN-SREBP-1 or $\beta$-gal. I IACh was determined as described in Figure $1 \mathrm{~A}$ and the I-V relationship plotted. Points are the mean of 7 determinations in 3 independent cultures. The decrease in current density was significant; $P<0.03$.

LPDS (compare Figures 1B and 4D). However, adenoviral expression of DN-SREBP-1 decreased $\mathrm{I}_{\mathrm{KACh}}$ to $-32.4 \pm 7 \mathrm{pA} / \mathrm{pF}$ (Figure 4D; $n=7, P<0.03)$. Comparison with $\mathrm{I}_{\mathrm{KACh}}$ in cells cultured in FBS $(-23 \pm 4 \mathrm{pA} / \mathrm{pF}$; Figure 1B) demonstrated that overexpression of DN-SREBP-1 reversed LPDS stimulation of $\mathrm{I}_{\mathrm{KACh}}$ by $75 \%$.

Mice with targeted disruption of SREBP-1 demonstrate a blunted response of the heart to parasympathetic stimulation. In order to determine the physiologic significance of SREBP- 1 regulation of $\mathrm{I}_{\mathrm{KACh}}$ activity and GIRK1 expression, we studied the parasympathetic response of the heart in SREBP-1 KO mice (18). SREBP-1 KO mice were challenged with carbamylcholine and heart rate determined using implantable ECG monitors. Animals were pretreated with propranolol in order to block $\beta$-adrenergic-stimulated reflex responses to carbamylcholine. Resting heart rates prior to propranolol treatment were not significantly different in WT and SREBP-1 KO mice: $594 \pm 20.7$ vs. $616.1 \pm 16.8 \mathrm{bpm}( \pm \mathrm{SEM}, n=7)$, respectively. Furthermore, there was no significant difference in the response to propranolol; heart rate decreased to $548.4 \pm 7.2$ and $548.0 \pm 6.6 \mathrm{bpm}$ in WT and SREBP-1 KO mice, respectively (Figure 5A). However, response of SREBP-1 KO mice to carbamylcholine was significantly decreased compared with WT. The maximum decrease in heart rate in response to carbamylcholine was $298.3 \pm 10.5 \mathrm{bpm}$ or $55.0 \% \pm 2.0 \%$ in WT compared with $263.9 \pm 11.2 \mathrm{bpm}$ or $48 \% \pm 2.0 \%$ for SREBP- $1 \mathrm{KO}$ mice $(n=7 ; P<0.04)$. The duration of bradycardia was decreased almost 2-fold in the SREBP-1 KO mice, $5.6 \pm 1.7 \mathrm{~min}$ vs. $10.5 \pm 1.3 \mathrm{~min}(P<0.05)$ in WT mice (Figure 5, A and B). Furthermore, following carbamylcholine treatment, heart rate recovered to $80 \%$ of pretreatment levels $\left(\operatorname{Rec}^{80}\right)$ in $5.23 \pm 0.79 \mathrm{~min}$ in SREBP-1 KO mice compared with $10.9 \pm 1.62$ min for WT mice $(P<0.025)$. These data indicate that deficiency of SREBP-1 was associated with a marked decrease in the response of the heart to parasympathetic stimulation compared with WT mice.

GIRK1 expression is decreased in atria of SREBP-1 KO mice. Extracts of liver and adipose tissue of SREBP-1 KO mice have been shown not to express SREBP-1 (18). Western blot analysis demonstrated the absence of SREBP-1 in atrial homogenates of SREBP-1 KO mice, while SREBP-2 expression was unchanged or slightly increased compared with WT (Figure 5C). If the expression of GIRK1 were regu- 
A

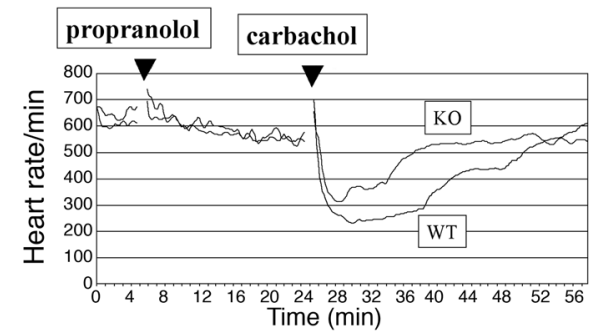

\section{B}

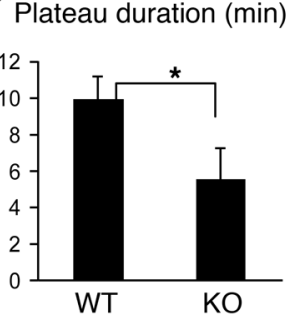

$80 \%$ recovery (min)
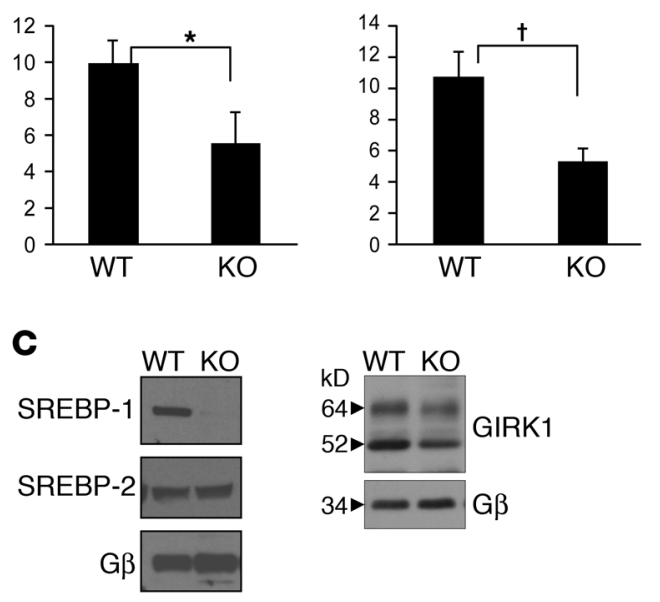

D

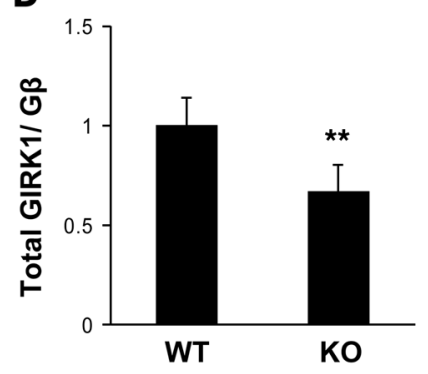

lated by SREBP, then GIRK1 expression might be decreased in atria of SREBP-1 KO mice and this decrease in GIRK1 expression might play a role in the decreased parasympathetic response. Western blot analysis of membrane extracts of atria from WT and SREBP-1 KO mice demonstrated 2 bands that had been previously shown to correspond to the glycosylated and unglycosylated forms of GIRK1 (19). Levels of both forms of GIRK1 were decreased in SREBP-1 KO mice (Figure 5C). Densitometry scanning of replicate determinations of GIRK1 expression demonstrated that total GIRK1 decreased by $38 \% \pm 7 \%$ in SREBP-1 KO mice compared with WT mice (Figure 5D). Thus, decreased SREBP-1 expression in atria of the SREBP-1 KO mice was associated with a decrease in GIRK1 expression.

Decreased $I_{K A C h}$ in atrial myocytes of SREBP-1 KO mice is reversed by adenoviral expression of SREBP-1. To determine whether the decrease in GIRK1 expression in atria of SREBP-1 KO mice is associated with a decrease in $\mathrm{I}_{\mathrm{KACh}}$, peak inward currents were compared in dissociated atrial myocytes for WT and SREBP-1 KO mice. $\mathrm{I}_{\mathrm{KACh}}$ decreased $54 \%$ from $-413 \pm 33 \mathrm{pA} / \mathrm{pF}(n=7)$ in WT hearts to $-192 \pm 21 \mathrm{pA} / \mathrm{pF}( \pm \mathrm{SEM}, n=7, P<0.001$; Figure 6 , A and $\mathrm{B})$ in cells from SREBP-1 KO hearts.

\section{Figure 5}

Response of the heart to parasympathetic stimulation and the expression of GIRK1 in SREBP-1 KO mice. (A) Comparison of the negative chronotropic response of WT and SREBP-1 KO mice to carbamylcholine. Continuous ECGs were recorded as described in Methods. Mice were treated with an intraperitoneal injection of propranolol, $1 \mathrm{mg} / \mathrm{kg}$, and 20 minutes later by intraperitoneal injection of carbachol, $0.2 \mathrm{mg} / \mathrm{kg}$. Data represent the mean heart rate of 7 age-matched mice. (B) Quantitation of the negative chronotropic response to carbachol in WT and SREBP-1 KO mice. The plateau time of bradycardia and the time elapsed for $80 \%\left(\mathrm{Rec}^{80}\right)$ recovery to baseline heart rate, defined as heart rate following propranolol treatment, were determined as described in Methods. ${ }^{*} P<0.05 ; \uparrow P<0.05$. (C) Expression of SREBP-1 and GIRK1 in atria of WT and SREBP-1 KO mice. Atria of age-matched male WT and SREBP-1 KO mice were harvested and homogenized, and SREBP-1 (left panel) and GIRK1 (right panel) were determined by Western blot analysis. (D) Mean intensity of both glycosylated and unglycosylated GIRK1 bands determined by densitometry analysis of autoradiographs from 8 WT and 8 SREBP-1 KO mice normalized to the expression of $\mathrm{G} \beta$; ${ }^{*} P<0.03$.

If the attenuation of $\mathrm{I}_{\mathrm{KACh}}$ in dissociated atrial myocytes from SREBP-1 KO mice is due to the absence of SREBP-1, then overexpression of SREBP-1 might rescue the phenotype and restore $\mathrm{I}_{\mathrm{KACh}}$ to levels seen in cells from WT mice. We developed conditions for maintaining viable adult mouse atrial myocytes in culture for up to 48 hours. Infection of these cells at the time of plating with an adenoviral vector expressing GFP resulted in GFP fluorescence in $85 \%$ of myocytes (data not shown). The peak inward currents in atrial myocytes from SREBP-1 KO mice infected for 48 hours with an adenovirus expressing GFP and $\beta$-gal was $-158 \pm 35 \mathrm{pA} / \mathrm{pF}$ (Figure 6C; $n=7$ ), which was not significantly different from that in uninfected cells (Figure 6B). Peak inward currents in atrial myocytes from hearts of SREBP-1 KO mice infected with adenovirus coexpressing GFP and SREBP-1 were $-317 \pm 27 \mathrm{pA} / \mathrm{pF}$ (Figure 6, $\mathrm{C}$ and $\mathrm{D} ; n=7), 2$-fold higher than atrial myocytes from control SREBP-1 KO mice expressing GFP and $\beta$-gal $(P<0.004)$. Thus, overexpression of SREBP-1 in atrial myocytes from SREBP-1 KO mice resulted in an increase of $\mathrm{I}_{\mathrm{KACh}}$ to $77 \%$ of peak currents measured in atrial myocytes from WT mice (Figure 6D).

SREBP-1 KO mice demonstrate an increased incidence of inducible ventricular arrhythmias following MI. Parasympathetic stimulation of the heart has been shown to be protective against the development of life-threatening ventricular arrhythmias, especially in the setting of MI $(1,20)$. Spontaneous arrhythmias during the acute phase of MI, especially during the first 6 hours, contribute to the high incidence of out-of-hospital sudden death. Early arrhythmias have been shown to have little long-term prognostic significance (21). However, the development of spontaneous or inducible ventricular arrhythmias during the chronic phase of MI has been associated with decreased survival (14). Studies of MI in the mouse heart have demonstrated that the healing phase is usually complete 2 weeks after infarction $(22,23)$. To determine whether the decreased parasympathetic responsiveness of the heart in the SREBP-1 KO mice is associated with a predisposition to the development of ventricular arrhythmias, the inducibility of ventricular arrhythmias in response to programmed stimulation of the ventricle was compared in WT and SREBP-1 KO mice 2 weeks following MI. The effect of SREBP-1 $\mathrm{KO}$ on the cardiac phenotype was determined by comparing chamber size and LV function in WT and SREBP-1 KO mice both in the basal state and following MI. There was no significant difference 
A

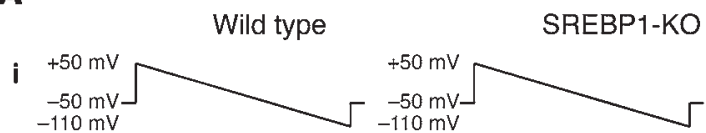

ii

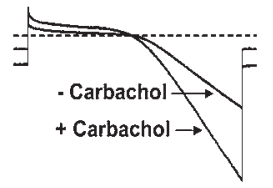

ií
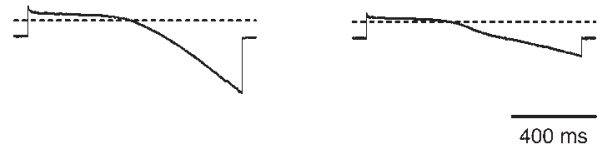

B

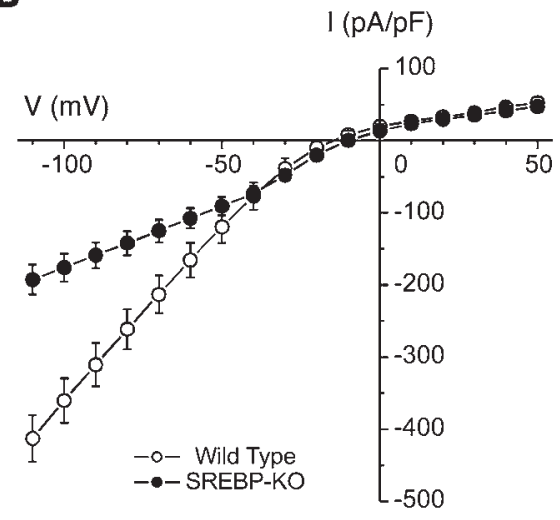

D

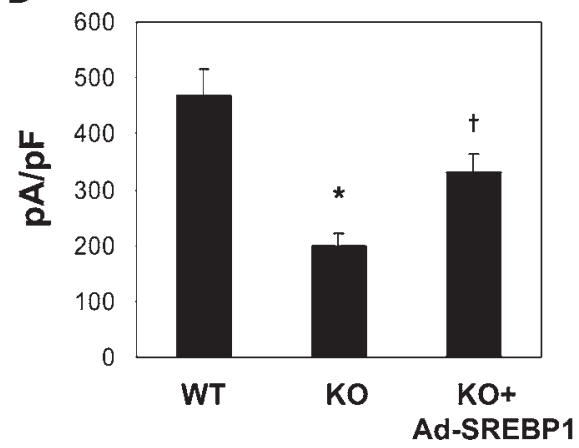

Figure 6

$I_{\text {KACh }}$ is markedly decreased in atrial myocytes from SREBP-1 KO mice compared with WT and increased by overexpression of SREBP-1c. (A) $\mathrm{I}_{\mathrm{KACh}}$ is decreased in dissociated atrial myocytes from SREBP-1 KO mice compared with cells from atria of WT mice, as described in Methods. $I_{\text {KACh }}$ was determined as described in Figure 1A. (B) I-V plots constructed from a series of data points in A, panel iii. Data represent the mean of 7 determinations from 3 separate preparations. (C) I-V plots from dissociated myocytes from atria of SREBP-1 KO mice infected with an adenovirus expressing GFP or GFP plus SREBP-1C (MOI of 20). Forty-eight hours after infection, IKACh was determined in GFP+ cells by fluorescence microscopy ( $90 \%$ of cells) as in A and B. Points are the mean of 7 determinations from 3 separate cultures. (D) Mean peak inward current from 7 determinations; ${ }^{*} P<0.001$ compared with $\mathrm{WT} ;{ }^{\dagger} P<0.004$ compared with $\mathrm{KO}$.

in end-diastolic dimension, end-systolic dimension, anterior and posterior wall thickness, fractional shortening, and basal heart rate between WT and SREBP-1 KO mice (Table 1).

Since differences in inducibility of VT following MI in WT and SREBP-1 KO mice might be due to differences in infarct size, LV dysfunction, or electrophysiologic properties, these parameters were compared in WT and SREBP-1 KO mice following MI. Infarct size measured echocardiographically was $34.0 \% \pm 1.3 \%$ in SREBP-1 KO mice $(n=11)$ and $35.7 \% \pm 2.7 \%(n=9, \mathrm{NS})$ in WT mice. Infarct size measured by planimetry of photomicrographs of the total area of the ventricle and the infarct zone was $30.7 \% \pm 3.1 \%(n=6)$ in WT mice and $31.5 \% \pm 3.2 \%(n=6, \mathrm{NS})$ in SREBP-1 KO mice. Hence infarct size was not significantly different in WT and SREBP-1 KO mice independent of the method of measurement. Furthermore, within each group, infarct size determined by either method was not significantly different.

As expected, end-diastolic diameter and end-systolic diameter increased in both WT and SREBP KO mice following MI. However, there was no significant difference in the magnitude of these changes in WT and SREBP-1 KO mice (Table 1). Furthermore, although fractional shortening decreased in both WT and SREBP-1
$\mathrm{KO}$ mice following MI, there was no significant difference in the magnitude of decrease in WT and SREBP-1 KO mice. Furthermore, ventricular wall thickness in the uninfarcted area of the anterior and posterior walls of the LV in WT and SREBP-1 KO mice was unaffected by MI. Finally, there were no statistically significant differences in RR, PR, QRS, QT, and QTc intervals and ventricular effective refractory period (VERP) in SREBP-1 KO mice compared with WT following MI (Table 2).

Inducibility of ventricular arrhythmias in response to programmed stimulation of the ventricle was compared in $7 \mathrm{WT}$ and 9 SREBP-1 KO mice 2 weeks following MI. All 16 animals survived surgery and the 14 day recovery period. Eight of 9 SREBP-1 KO mice demonstrated inducible VT as compared with 3 of 7 WT mice ( $89 \%$ vs. $43 \%, P<0.05$; Figure 7 A). Figure $7 \mathrm{~B}$ demonstrates a typical inducible response in an SREBP-1 KO mouse following programmed stimulation with a sensed triple extrastimulus.

\section{Discussion}

The balance between sympathetic and parasympathetic stimulation of the heart has been shown to play a protective role in the development of arrhythmias and sudden death (1). Here, we 
Table 1

Contractile function of SREBP-1 KO mice

\begin{tabular}{lcccc} 
& \multicolumn{2}{c}{ Before MI } & \multicolumn{2}{c}{ After MI } \\
& SREBP-1 KO & WT & SREBP-1 KO & WT \\
EDD & $3.22 \pm 0.12$ & $3.16 \pm 0.17$ & $4.16 \pm 0.20$ & $4.05 \pm 0.29$ \\
ESD & $2.80 \pm 0.12$ & $2.04 \pm 0.19$ & $3.09 \pm 0.19$ & $3.04 \pm 0.31$ \\
Post wall & $1.03 \pm 0.06$ & $1.03 \pm 0.03$ & $1.04 \pm 0.05$ & $0.97 \pm 0.03$ \\
Ant wall & $1.05 \pm 0.03$ & $1.00 \pm 0.04$ & $0.99 \pm 0.02$ & $1.01 \pm 0.02$ \\
FS & $44.1 \pm 2.86$ & $38.0 \pm 2.82$ & $26.0 \pm 1.27$ & $25.7 \pm 2.93$ \\
HR & $410 \pm 10$ & $400 \pm 20$ & $443 \pm 28$ & $440 \pm 20$
\end{tabular}

SREBP-1 KO $(n=6)$ and WT mice $(n=6)$. EDD, end-diastolic diameter $(\mathrm{mm})$; ESD, end-systolic diameter $(\mathrm{mm})$; post wall, posterior wall thickness $(\mathrm{mm})$; ant wall, anterior wall thickness ( $\mathrm{mm})$; FS, fractional shortening (\%); $\mathrm{HR}$, heart rate $(\mathrm{bpm})$. No difference was found statistically significant.

demonstrate what we believe is a novel relationship between lipid homeostasis and the regulation of the signaling pathway through which parasympathetic stimulation via the central nervous system regulates the rate of contraction of the heart. We further demonstrate that the disruption of the pathway for the regulation of lipid homeostasis results in a decrease in parasympathetic responsiveness of the heart and the predisposition of the mouse heart to the development of ventricular arrhythmias, suggesting a new relationship between lipid metabolism and arrhythmogenesis.

Parasympathetic signaling and expression of $M_{2}, G \alpha_{i 2}$, and GIRK1. The parasympathetic response of the heart involves the binding of acetylcholine to the $\mathrm{M}_{2}$-muscarinic receptor and its interaction with the heterotrimeric $\mathrm{G}$ protein $\mathrm{G}_{\mathrm{i} 2}$ and the inward-rectifying $\mathrm{K}^{+}$channel, resulting in the stimulation of $\mathrm{I}_{\mathrm{KACh}}(5)$. Overexpression of $\mathrm{Go}_{\mathrm{i} 2}$ in the porcine $\mathrm{AV}$ node (24) has been shown to decrease $\mathrm{AV}$ nodal conduction. Developmental studies have demonstrated that the appearance of a negative chronotropic response to parasympathetic stimulation is associated with an increase in $\mathrm{G \alpha}_{\mathrm{i} 2}$ expression in chick atrial myocytes (25). Furthermore, TGF- $\beta$ has been shown to regulate the parasympathetic response of the heart via an effect on $\mathrm{G \alpha}_{\mathrm{i} 2}$ and $\mathrm{M}_{2}$ expression $(26,27)$. These studies support the conclusion that the parasympathetic response of the heart may be regulated by changes in expression of parasympathetic response genes.

The role of SREBP-1 in the transcriptional regulation of GIRK1 expression. Lipid lowering is a major therapeutic end point in the treatment of atherosclerosis. The finding that GIRK1 expression might be regulated by lipid metabolism was first suggested by studies in an in vitro model for lipid lowering in which chick ventricular myocytes were cultured in LPDS. Lipoprotein depletion resulted in an increase in the negative chronotropic response to carbamylcholine and in the level of mRNA coding for GIRK1 $(6,7)$. Subsequently it was demonstrated that lipid lowering regulated expression of $\mathrm{G \alpha}_{\mathrm{i} 2}$ in chick atrial myocytes by binding of SREBP-1 to a single SRE in the $\mathrm{G}_{\mathrm{i} 2}$ promoter (13).

In the current study, the finding that lipid lowering by growth of atrial myocytes in LPDS increased both the expression of GIRK1 and GIRK1 promoter activity further supported the role of lipids in the regulation of GIRK1 expression. The finding that lipid lowering increased SREBP levels in these cells (13) was consistent with the hypothesis that SREBP might regulate GIRK1 expression. Furthermore, reporter assays demonstrated that SREBPs stimulated GIRK1 promoter activity in a dose-dependent manner, which was specific for SREBP-1a and -1c, and not SREBP-2 (17).
Finally, both LPDS and SREBP-1 stimulation of GIRK1 promoter activity were abolished in promoter constructs in which a site containing 2 putative tandem SREs and an upstream E-box had been deleted. Furthermore, point mutations in both SRE-1 and SRE-2 appeared to have an additive effect on inhibition of SREBP-1 and LPDS regulation of the GIRK1 promoter. However, only mutagenesis of both SREs and the E-box abolished stimulation of GIRK1 promoter activity by either LPDS or SREBP-1. In contrast, lipid lowering and SREBP-1 regulated $\mathrm{G} \alpha_{\mathrm{i} 2}$ expression via a single SRE in the $\mathrm{G}_{\mathrm{i} 2}$ promoter (13). Hence, both SREBP-1 and LPDS stimulation of the GIRK1 promoter were dependent on a similar set of cis-acting elements.

SREBP-1 regulation of $I_{\text {KACb. }}$. The role of SREBP- 1 in the regulation of GIRK1 expression was further supported by the finding that adenoviral expression of DN-SREBP-1 in atrial myocytes grown in LPDS reversed the effect of LPDS on GIRK1 expression, while adenoviral expression of SREBP-1 in atrial myocytes cultured in FBS stimulated GIRK1 protein expression to levels similar to those found in cells cultured in LPDS. These data strongly support the conclusion that SREBP-1 regulates the expression of GIRK1 in response to lipid lowering and that GIRK1 expression is regulated at least in part by sterols.

The relationship among SREBP-1 signaling, GIRK1 expression, and the response of atrial myocytes to parasympathetic stimulation is supported by the finding that overexpression of DN-SREBP-1 reversed the stimulatory effects of LPDS on $\mathrm{I}_{\mathrm{KACh}}$ in cultured chick atrial myocytes.

Parasympathetic responsiveness in the SREBP-1 KO mouse and GIRK1 expression. The availability of the SREBP-1 KO mouse provided an animal model to test the role of SREBP- 1 in the regulation of the parasympathetic response in vivo. Intraperitoneal injection of carbamylcholine directly stimulates the heart, bypassing centrally mediated parasympathetic input from the vagus nerve. Hence, it constitutes a direct measure of the responsiveness of the heart to parasympathetic signaling and the intactness of the intrinsic molecular cardiac signaling mechanism for parasympathetic regulation in the heart. Compared with WT animals, hearts of the SREBP-1 KO mice demonstrated a marked decrease in response to carbamylcholine: a decrease in the duration of bradycardia and a more rapid recovery of the heat rate to baseline in response to carbamylcholine. Interestingly, this correlated with the nearly $40 \%$ decrease in the level of expression of GIRK1 in the atria of SREBP-1 KO mice compared with WT.

Viral expression of SREBP-1 restores $I_{K A C b}$ in atrial myocytes from SREBP-1 KO mice. $\mathrm{I}_{\mathrm{KACh}}$ was decreased more than 2-fold in atrial myocytes of SREBP-1 KO mice. The relationship between the loss

\section{Table 2}

Electrophysiologic parameters of SREBP-1 KO mice

$\begin{array}{lcc} & \text { SREBP-1 } \mathbf{K O}(\boldsymbol{n}=\mathbf{9}) & \text { WT }(\boldsymbol{n}=\mathbf{7}) \\ \text { RR } & 122 \pm 3 & 118 \pm 7 \\ \text { PR } & 44 \pm 1 & 47 \pm 3 \\ \text { QRS } & 14 \pm 1 & 16 \pm 2 \\ \text { QT } & 55 \pm 3 & 58 \pm 2 \\ \text { QTC } & 50 \pm 2 & 53 \pm 2 \\ \text { VERP } & 21 \pm 4 & 25 \pm 2\end{array}$

Units of all parameters are in ms. No difference was found to be statistically significant. 
A
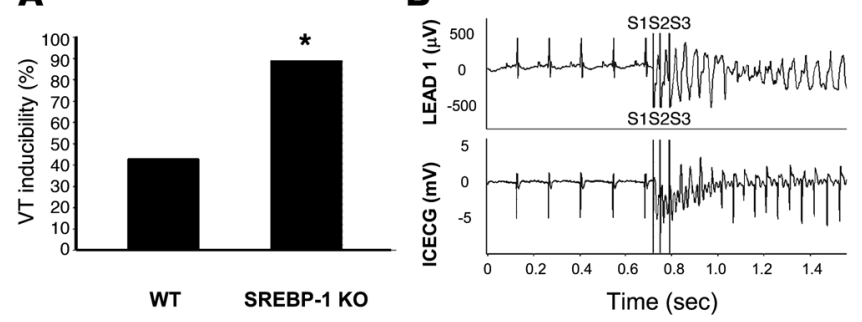

Figure 7

Inducibility of ventricular arrhythmias in WT and SREBP-1 KO mice following MI. To generate Mls, the left coronary artery was ligated in 9 SREBP-1 KO and 7 WT mice. For programmed ventricular stimulation, a 1.1-Fr octapolar catheter was inserted into the right ventricle via the right external jugular vein until electrograms were obtained as described in Methods. Surface ECG leads were obtained using 24-gauge needles placed subcutaneously in each limb. (A) Inducibility of VT in WT and SREBP-1 KO mice in response to programmed stimulation. Data represent the percentage of mice in each group that developed VT; ${ }^{*} P<0.05$. (B) Typical response of an SREBP-1 KO mouse to programmed ventricular stimulation. Simultaneous recordings from the surface ECG (lead I) and an intracardiac ECG (ICECG) are shown. The stimulation protocol consisted of sensed programmed single, double, and triple extrastimuli (S1, S2, and S3). A positive study was defined as 7 or more beats of VT.

of SREBP-1 and the decreased parasympathetic response in the SREBP-1 KO mouse was most directly demonstrated by the finding that viral expression of SREBP-1 increased $\mathrm{I}_{\mathrm{KACh}}$ in atrial myocytes from SREBP-1 KO mice to levels similar to those in cells from WT mice. Given that the effects of carbamylcholine on both $\mathrm{I}_{\mathrm{KACh}}$ and the negative chronotropic response of the heart to parasympathetic stimulation are independent of vagal innervation, these data demonstrate that SREBP-1 plays a role in the regulation of the intrinsic parasympathetic signaling pathway in the heart.

Inducibility of ventricular arrbythmias following MI in SREBP-1 KO mice. The relationship among vagal activity, parasympathetic stimulation, and the incidence of life-threatening arrhythmias is supported by data from multiple sources including a canine "exercise and ischemia" model for ventricular fibrillation (VF) in which atropine increased the incidence of VF (1) and direct muscarinic activation by electrical stimulation of the left vagus nerve protected animals from the development of VF (28). In patients, sudden death has been associated with a decrease in the high-frequency component of heart rate variability, increased dispersion of QT intervals, and decreased rate of return of heart rate to baseline after exercise (29, $30)$. All of these have been associated with impaired parasympathetic responsiveness of the heart. Furthermore, more than $50 \%$ of patients with a 10-year history of diabetes demonstrate an impaired response of the heart to parasympathetic stimulation and a resulting sympathovagal imbalance (31), or DAN. The presence of DAN is associated with a 5-fold higher 5-year mortality compared with diabetics without DAN (32). Finally, the incidence of sudden death was 2.8 times higher in diabetics compared with normoglycemic patients (3). Thus, regulation of the balance between sympathetic and parasympathetic response of the heart plays an important role in the incidence of sudden death, especially in the setting of MI.

In patients, the period following MI has been divided into 3 phases: an acute phase lasting 48 hours, characterized by apoptosis and cell death; a healing phase, from 48 hours to 6 weeks; and a chronic phase, which begins at 6 weeks and is characterized by ventricular remodeling. Primary ventricular arrhythmias occurring during the acute phase, especially within the first 6 hours, are associated with sudden death. Survivors of ventricular arrhythmias occurring during the first 48 hours have the same long-term survival as patients without ventricular arrhythmias in the acute phase (21). During the healing phase, episodes of nonsustained VT have no prognostic significance, while episodes of sustained VT do. However, during the chronic or remodeling phase, both sustained and nonsustained episodes of VT and inducibility of VT by programmed ventricular stimulation are associated with an increased incidence of life-threatening arrhythmias and decreased survival $(14,15,33)$. Ventricular healing following MI in the mouse has been shown to occur over the first 2 weeks after MI (23). We compared the inducibility of ventricular arrhythmias in the SREBP-1 KO mouse in response to programmed ventricular stimulation 2 weeks after MI. Following MI, there were no significant differences in infarct size, end-diastolic ventricular volume, end-systolic ventricular volume, percentage fractional shortening, QRS duration, or QTc intervals in SREBP-1 KO mice compared with WT. Finally, VERP, which has been shown to be associated with increased inducibility of VT and VF (34), was not significantly prolonged in SREBP-1 KO mice compared with WT mice. Thus, the finding of increased inducibility of VT in SREBP-1 KO mice supports the conclusion that SREBP-1 might protect the heart from the development of arrhythmias via an effect on parasympathetic signaling.

The clinical significance of these findings is supported by data demonstrating that lipid lowering in patients treated with HMGCoA reductase inhibitors resulted in a significant increase in the parasympathetic responsiveness of the heart, which correlated with a marked increase in the expression of $\mathrm{G \alpha}_{\mathrm{i} 2}$ in lymphocytes of these patients (32). Furthermore, retrospective studies of the effect of lipid lowering by HMG-CoA reductase inhibitors on the incidence of ventricular arrhythmias, as measured by firing of implantable defibrillators in patients with coronary artery disease and nonischemic cardiomyopathy, support the conclusion that lipid lowering by HMG-CoA reductase inhibitors might protect the heart from the development of ventricular arrhythmias (35, 36). Thus, in analysis of data from the Multicenter Autonomic Defibrillator Implantation Trial (MADIT) II, statin use in patients with an implantable cardioverter defibrillator (ICD) was associated with a decrease in the end points of VT/VF or cardiac death (35). In a group of 78 patients with coronary artery disease and ventricular arrhythmias treated with an ICD, 6 of 27 , or $22 \%$, of patients treated with lipid-lowering drugs demonstrated a decreased incidence of ventricular arrhythmias while 29 of 51 , or $57 \%$, of patients not treated with lipid-lowering drugs did not (37). The interpretation of these studies has been complicated by the presence of ischemic heart disease. However, retrospective analysis of patients in the DEFIbrillators in Non-Ischemic cardiomyopathy Treatment Evaluation (DEFINITE) study suggested that statin use was associated with a $78 \%$ decrease in mortality, which was in part due to a reduction in arrhythmic death (36). Thus, SREBP-1 regulation of GIRK1 expression and $\mathrm{I}_{\mathrm{KACh}}$ might constitute a new relationship between parasympathetic function and lipid metabolism and a novel mechanism for regulation of autonomic responsiveness that might play an important role in the pathogenesis and treatment of autonomic dysfunction and the genesis of life-threatening ventricular arrhythmias. 


\section{Methods}

Materials. Ad-SREBP-1c (ADD-1) was a gift from Bruce Spiegelman (DanaFarber Cancer Center, Harvard Medical School, Boston, Massachusetts, USA). Ad-GFP was a kind gift from Anthony Rosenzweig (Beth Israel Deaconess Medical Center, Harvard Medical School, Boston, Massachusetts, USA). The SREBP-1 KO mouse has been described previously (18). C57BL/6J WT mice were obtained from the SREBP-1 KO colony littermates (18). The GIRK1 antibody was from Alomone Labs. LPDS was prepared as described previously (38).

Cell culture. Embryonic chick atrial myocytes were cultured as described (13). For electrophysiological studies, atrial myocytes were plated on glass coverslips precoated with $2 \%$ gelatin (Sigma-Aldrich). Dissociated atrial myocytes from mouse atria were prepared by a retrograde Langendorff perfusion method. The heart was placed in cold saline and the aorta cannulated and perfused with $\mathrm{Ca}^{2+}$-free Tyrode buffer, $10 \mathrm{mM}$ glucose, $10 \mathrm{mM}$ 2,3-butanedione monoxime (BDM) (Sigma-Aldrich), and $5 \mathrm{mM}$ taurine ( $\mathrm{pH} 7.4$; Sigma-Aldrich) via a Langendorff apparatus at a rate of $3 \mathrm{ml} / \mathrm{min}$ at $37^{\circ} \mathrm{C}$ for 3 minutes followed by a $7-10$ minute perfusion at the same rate with an enzyme solution containing collagenase $B(0.4 \mathrm{mg} / \mathrm{g}$ body weight; Roche), collagenase D (0.3 mg/g; Roche), and proteinase XIV (0.05 mg/g; Sigma-Aldrich) in $25 \mathrm{ml}$ of perfusion buffer. Atria were dissected away, placed in plating medium, and cut into small pieces followed by several rounds of pipetting to dissociate cells. Cells were plated in myocyte plating medium (MEM [GIBCO], $100 \mathrm{U} / \mathrm{ml}$ penicillin, $2 \mathrm{mM}$ L-glutamine, 5\% FBS, $10 \mathrm{mM} \mathrm{BDM}$, and $10 \mu \mathrm{g} / \mathrm{ml}$ ascorbate) onto laminin-coated glass coverslips (10 $\mu \mathrm{g} / \mathrm{ml}$, Invitrogen) and incubated in $2 \% \mathrm{CO}_{2}$ for 1 hour to allow attachment to the coverslip. Cells were rod shaped with clearly defined striations and contracted spontaneously.

Plasmid constructs. The p30-Luc-MTI vector containing the human GIRK1 promoter linked to luciferase, which was used for mutagenesis, has been previously described (39). Point mutations were introduced in the SRE and E-box sites of the GIRK1 promoter by site-directed mutagenesis using the QuikChange Site-Directed Mutagenesis Kit (Stratagene). To create the p30FX-Luc deletion mutant, the FbaI/XhoI region (-1258 to -1010), which contains the 2 putative SRE sites, was deleted from the GIRK1 promoter, followed by fill-in with Klenow polymerase and blunt-end ligation. All other deletion mutants of the GIRK1 promoter have been previously described (39). To create the E-SRE-Luc construct, the promoter region from -1297 to -1005 was generated by PCR and subcloned in the SacI/XhoI sites of pGL2, which contains the minimal thymidine kinase promoter cloned into BglII/HindIII sites. All constructs were verified by restriction enzyme digestion and DNA sequencing.

Transient transfection and reporter assays. All transfections were carried out using LipofectAMINE transfection reagent (Invitrogen) according to the manufacturer's instructions. For GIRK1-luciferase assays, chick embryo atrial myocytes were cultured in 12-well plates. Typically, transfections contained $150 \mathrm{ng}$ GIRK1 promoter reporter, $5 \mathrm{ng}$ pCMV- $\beta$-gal, and $100 \mathrm{ng}$ of the SREBP-expressing plasmid. To maintain total DNA constant at $1 \mu \mathrm{g} /$ well, pBluescript vector was used. For LPDS experiments, atrial myocytes were plated in either 6\% FBS or an equivalent protein amount of LPDS. Typically, cells were transfected on the second culture day. On the third culture day, cells were incubated in fresh media and incubation continued for an additional 24 hours prior to harvest. Cells were lysed in Reporter Lysis Buffer (Promega) and assayed for luciferase (Luciferase Assay System; Promega) and $\beta$-gal activity (Chemiluminescent Reporter Gene Assay System; Applied Biosystems). Luciferase units were normalized to $\beta$-gal activity, which served as an internal control for transfection efficiency.

Western blot analysis. To determine GIRK1 levels in cultured atrial myocytes, cells were harvested and the membrane fractions prepared as follows: cells were resuspended in $1 \mathrm{ml}$ of a hypotonic buffer $(10 \mathrm{mM}$ HEPES, pH 7.4,
$10 \mathrm{mM} \mathrm{KCl}, 1.5 \mathrm{mM} \mathrm{MgCl}_{2}, 0.1 \mathrm{mM}$ EDTA, $0.1 \mathrm{mM}$ EGTA, $1 \mathrm{mM}$ DTT, $0.5 \mathrm{mM}$ PMSF, 1:100 diluted Sigma-Aldrich protease inhibitor cocktail) and sonicated by using a Branson Sonifier 450. The cell suspension was centrifuged at $1000 \mathrm{~g}$ in a microfuge, and the supernatants centrifuged in a Beckman ultracentrifuge at $100,000 \mathrm{~g}$ for 40 minutes. The membrane fractions in the pellet were solubilized in an SDS-containing buffer $(50 \mathrm{mM}$ Tris $\mathrm{Cl}$, pH 7.4, 0.15 M NaCl, 1.5 mM MgCl 2,1 mM EDTA, 1 mM EGTA, 0.5\% SDS, $1 \mathrm{mM}$ DTT, $0.5 \mathrm{mM}$ PMSF, 1:100 diluted Sigma-Aldrich protease inhibitor cocktail). In order to determine the levels of GIRK1 expression in atrial tissues of SREBP-1 KO and WT mice, tissues were homogenized by using a Dounce homogenizer in $1 \mathrm{ml}$ of the hypotonic buffer and the membrane fractions prepared as above. The concentration of proteins was determined by using a Bradford reagent (Bio-Rad). Equal amounts of membrane proteins were analyzed to determine the levels of GIRK1 by Western blot analysis using a GIRK1 antibody from Alomone Labs. In order to determine the levels of SREBP expression in atrial tissues of SREBP-1 KO and WT mice, whole tissue extracts were prepared by using a Triton $\mathrm{X}-100$-containing buffer (50 mM Tris Cl, pH 7.4, 0.15 M NaCl, $1.5 \mathrm{mM} \mathrm{MgCl}_{2}, 1 \mathrm{mM}$ EDTA, $1 \mathrm{mM}$ EGTA, 1\% Triton X-100, 1 mM DTT, 0.5 mM PMSF, 1:100 diluted Sigma-Aldrich protease inhibitor cocktail, and $25 \mu \mathrm{M} \mathrm{N}$-acetyl-leu-leu-norleucinal $[\mathrm{ALLN}])$. A rabbit polyclonal anti-SREBP-1 and a goat polyclonal anti-SREBP-2 antibody were from Santa Cruz Biotechnology Inc.

Recombinant adenovirus constructs. The DN-SREBP-1-expressing adenovirus was constructed by the method of He et al. (40). In brief, DN-SREBP-1c cDNA (41) was subcloned in the adenoviral shuttle vector (pAdTrack) under the control of a CMV promoter. This shuttle vector also contained a GFP under an independent CMV promoter. pAdEasy1, a backbone vector containing most of the adenoviral genome, was used together with the shuttle vector to create a recombinant adenovirus in Escherichia coli, which was propagated in HEK293 cells. As control, we used an adenovirus containing both $\beta$-gal and GFP under the control of separate CMV promoters.

Gel mobility shift assays. Synthetic oligonucleotides containing the tandem putative SRE sites or the E-box site of the human GIRK1 promoter used as probes in gel-shift analysis were as follows (point mutations are indicated in bold): probe for WT, 5'-CTGATCACACGCACCACCACACACACCACATTCCTGCTGCCCC-3'; S1, 5'-CTGATCACACGCACAACCACACACACCACATTCCTGCTGCCCC-3'; S2, 5'-CTGATCACACGCACCACCACACAAACCACATTCCTGCTGCCCC-3'; S1,2, 5'-CTGATCACACGCACAACCACACAAACCACATTCCTGCTGCCCC-3'; E-box, 5'-GCGCGGCTCTGCAGCTGATCACAC-3'; and E-box mutant, 5'GCGCGGCTCTGTGTAACATCACAC-3'. Each of these oligonucleotides was annealed to its complimentary strand, creating double-stranded probes with a 4-base overhang labeled with $[\alpha-32 \mathrm{P}]$-deoxy-CTP using Klenow DNA polymerase I (New England Biolabs). Binding reactions typically contained $0.1 \mathrm{ng}$ of probe DNA, $20 \mathrm{ng}$ of purified recombinant human mature SREBP-1a protein, prepared as previously described (42), and $1 \mu \mathrm{g}$ of poly-(dI-dC) $(\mathrm{dI}-\mathrm{dC})$ in a buffer containing $12 \mathrm{mM}$ HEPES ( $\mathrm{pH} 7.6)$, $1 \mathrm{mM} \mathrm{MgCl}_{2}, 50 \mathrm{mM} \mathrm{KCl}, 5 \%$ glycerol, and 4\% Ficoll 4000, in a total volume of $20 \mu \mathrm{l}$. For supershift experiments, $0.1 \mu \mathrm{g}$ of rabbit polyclonal SREBP-1 affinity-purified IgG (K-10; Santa Cruz Biotechnology Inc.) was used. Reactions were performed at room temperature for 30 minutes and proteinDNA complexes separated by electrophoresis on $5 \%$ polyacrylamide gel in $\times 0.5$ Tris-borate-EDTA buffer at $4^{\circ} \mathrm{C}$.

Cellular electrophysiology. Membrane currents were measured by the patchclamp technique in whole-cell mode using an LM-EPC7 amplifier. Immediately prior to seal formation, the extracellular solution was replaced with a high $\mathrm{K}^{+}$solution without $\mathrm{Ca}^{2+}$ to inhibit contraction: $100 \mathrm{mM} \mathrm{NaCl}, 50 \mathrm{mM}$ $\mathrm{KCl}, 1 \mathrm{mM} \mathrm{MgCl}$, $5 \mathrm{mM}$ HEPES, and $5.5 \mathrm{mM}$ D-glucose, adjusted to $\mathrm{pH}$ 7.4. Patch electrodes were pulled from $1.5-\mathrm{mm}$ diameter Fischer brand glass capillaries and demonstrated a $2 \mathrm{M} \Omega$ resistance when filled with 
pipette solution: $140 \mathrm{mM} \mathrm{KCl}, 1 \mathrm{mM} \mathrm{MgCl} 2,10 \mathrm{mM}$ HEPES, 5 mM EGTA, $5 \mathrm{mM} \mathrm{Mg}_{2} \mathrm{ATP}$, and $0.1 \mathrm{mM}$ GTP, pH adjusted to 7.2 with KOH. Data were acquired by an Axon Instrument Digitizer (Digidata 1322B) with pClamp 9 software. Whole cell currents were elicited as described (38) in the presence and absence of $20 \mu \mathrm{M}$ carbamylcholine introduced by focal perfusion over $10-15$ seconds followed by washout. Currents returned to baseline within 10-15 seconds of washout. In order to correct for differences in cell size, currents were normalized to the cell capacitance determined via capacitance compensation and data presented as current density in $\mathrm{pA} / \mathrm{pF}$. Current densities differed significantly between chick and mouse atrial myocytes. However, capacitance was not significantly different: $21.9 \pm 1.9 \mathrm{pF}( \pm$ SEM, $n=11)$ in the mouse and $25.6 \pm 1.6 \mathrm{pF}( \pm \mathrm{SEM}, n=11)$ in the chick.

ECG monitoring in conscious mice. An implantable wireless radio frequency transmitter was inserted into a subcutaneous tissue pocket, the cathode lead anchored with a suture over the right scapula, and the anode lead anchored with a second suture over the apex of the heart. Signals were recorded with the use of a telemetry receiver and an analog to digital acquisition system (Data Sciences International). Male, age-matched WT and SREBP-1 KO mice, 8-12 months old, were studied. Mice were injected with $1 \mathrm{mg} / \mathrm{kg}$ propranolol followed 20 minutes later by $0.2 \mathrm{mg} / \mathrm{kg}$ carbamylcholine, as indicated. Heart rate was recorded until it returned to baseline and beat-to-beat heart-rate data obtained from raw ECGs by R-wave peak detection. Lowest heart rate after carbamylcholine injection and the elapsed time until the initiation of recovery was defined as the plateau time of bradycardia. The recovery phase was characterized by the time elapsed for $80 \%\left(\operatorname{Rec}^{80}\right)$ recovery.

Electrophysiology studies and programmed ventricular stimulation. Two weeks after coronary ligation, in vivo mouse electrophysiology studies were performed as previously described (34). In brief, anesthesia was induced with $2.5 \%$ isoflurane in oxygen and maintained with $2.0 \%$ isoflurane in oxygen. A surface frontal plane ECG was obtained from 24-gauge electrodes placed subcutaneously in each limb. ECG intervals (RR, PR, QRS, and QT) were measured for each animal. The corrected QT interval was calculated: $\mathrm{QTc}=\mathrm{QT} /(\mathrm{RR})^{1 / 2}$. An octapolar 1.1F electrophysiology catheter $(\mathrm{EPR}-800$; Millar Instruments) was inserted via the jugular vein cut-down approach and used for recording intracardiac electrograms and ventricular stimulation. Ventricular stimulation was performed using a commercially available external stimulator (Medtronic). Standardized pacing protocols were used to define VERPs, and each mouse underwent an identical programmed stimulation protocol. The stimulation protocol for ventricular arrhythmia consisted of sensed programmed right ventricular single, double, and triple extrastimuli. Right ventricular burst pacing with rates up to $75 \mathrm{~ms}$ for up to 1 minute duration was also performed. A negative study was defined as no induction of ventricular beats or induction of up to 6 repetitive ventricular beats. A positive study consisted of 7 or more repetitive ventricular beats induced by programmed ventricular stimulation.

Echocardiography and determination of infarct size. Three days prior to coronary ligation and on days 1 and 14 after coronary ligation, echocardiographic studies were performed as previously described (43). In brief, a commercially available echocardiography system (Sonos 7500; Phillips Medical Systems) was utilized with a dynamically focused linear array transducer (15-6L Intraoperative Linear Array; Phillips Medical Systems) using a depth setting of $0.5-1.0 \mathrm{~cm}$. Anesthesia was induced with inhaled $2.5 \%$ isoflurane in oxygen and maintained with inhaled $2.0 \%$ isoflurane in oxygen. Animals were placed on a warming pad to maintain body temperature at $36.5^{\circ} \mathrm{C}$ to $37.5^{\circ} \mathrm{C}$. Two-dimensional images and M-mode tracings (sweep speed, $50-100 \mathrm{~mm} / \mathrm{s}$ ) were then recorded from the short-axis view at the papillary muscle level. Using M-mode tracings, LV end-diastolic diameter (EDD) and end-systolic diameter (ESD) were measured to the nearest $0.1 \mathrm{~mm}$, averaging 3 cardiac cycles. Fractional shortening (FS) was calculated using the standard equation: $(\mathrm{EDD}-\mathrm{ESD}) / \mathrm{EDD} \times 100$. For determination of infarct size, 4 serial end-diastolic frames were captured from apex to base. The length (endocardial portion) of the akinetic segment and total LV circumference were measured by planimetry from each image. Infarct size was then calculated as the sum of all akinetic lengths divided by the sum of all total LV circumference measurements. Infarct size was also determined by planimetry of photomicrographs of the ventricle and infarct. The heart was placed between 2 glass slides, and images of both sides were digitally captured by photomicrography. The relative area of the infarct was determined by planimetry of the total area of the ventricle and infarct zone determined from both anterior and posterior views of the heart. There was good correlation between infarct sizes determined by both methods. All vertebrate animal-related procedures described above were approved by the Institutional Animal Care Committee at Tufts-New England Medical Center.

Statistics. All values are expressed as mean \pm SEM. Statistical differences between mean values were calculated by ANOVA, followed by Bonferroni's test for pairwise comparisons where appropriate. $P \leq 0.05$ was considered significant.

\section{Acknowledgments}

This work was supported by NIH grant HL074876 (to J.B. Galper), an American Heart Association Scientist Development grant (to H.-J. Park), and a Juvenile Diabetes Research Foundation postdoctoral fellowship (to Y. Zhang). The cellular electrophysiology studies were carried out in the Tufts University Center for Neuroscience Research, supported by NIH grant P30 NS047243.

Received for publication March 2, 2007, and accepted in revised form October 3, 2007.

Address correspondence to: Jonas B. Galper or Ho-Jin Park, Molecular Cardiology Research Institute, Tufts-New England Medical Center, 750 Washington St., Boston, Massachusetts 02111, USA. Phone: (617) 636-9004; Fax: (617) 636-4833; E-mail: jgalper@ tufts-nemc.org (J.B. Galper). Phone: (617) 636-9005; Fax: (617) 636-4833; E-mail: hpark@tufts-nemc.org (H.-J. Park).

Ulrike Begley's present address is: Department of Biomedical Sciences, School of Public Health, University at Albany, State University of New York, Rensselear, New York, USA.

Ho-Jin Park and Serban P. Georgescu contributed equally to this work.
Circulation. 91:2591-2595

4. Logothetis, D.E., Kurachi, Y., Galper, J., Neer, E.J., and Clapham, D.E. 1987. The beta gamma subunits of GTP-binding proteins activate the muscarinic K+ channel in heart. Nature. 325:321-326.

5. Vivaudou, M., et al. 1997. Probing the G-protein regulation of GIRK1 and GIRK4, the two subunits of the KACh channel, using functional homomeric mutants. J. Biol. Chem. 272:31553-31560.

6. Haigh, L.S., Leatherman, G.F., O'Hara, D.S., Smith, T.W., and Galper, J.B. 1988. Effects of low density lipoproteins and mevinolin on cholesterol content and muscarinic cholinergic responsiveness in cultured chick atrial cells. Regulation of levels of muscarinic receptors and guanine nucleotide regulatory proteins. J. Biol. Chem. 263:15608-15618.

7. Gadbut, A.P., Toupin, D.K., Kilbourne, E.J., and Galper, J.B. 1994. Low density lipoproteins induce parasympathetic responsiveness in embryonic chick ventricular myocytes in parallel with a coordinate increase in expression of genes coding for the M2 muscarinic receptor, $\mathrm{G}$ alpha i2, and the 
acetylcholine-sensitive $\mathrm{K}+$ channel. J. Biol. Chem. 269:30707-30712.

8. Brown, M.S., Ye, J., Rawson, R.B., and Goldstein, J.L. 2000. Regulated intramembrane proteolysis: a control mechanism conserved from bacteria to humans. Cell. 100:391-398.

9. Matsuda, M., et al. 2001. SREBP cleavage-activating protein (SCAP) is required for increased lipid synthesis in liver induced by cholesterol deprivation and insulin elevation. Genes Dev. 15:1206-1216.

10. Anderson, R.G. 2003. Joe Goldstein and Mike Brown: from cholesterol homeostasis to new paradigms in membrane biology. Trends Cell Biol. 13:534-539.

11. Magana, M.M., and Osborne, T.F. 1996. Two tandem binding sites for sterol regulatory element binding proteins are required for sterol regulation of fatty-acid synthase promoter. J. Biol. Chem. 271:32689-32694.

12. Xiong, S., Chirala, S.S., and Wakil, S.J. 2000. Sterol regulation of human fatty acid synthase promoter I requires nuclear factor-Y- and $\mathrm{Sp}$-1-binding sites. Proc. Natl. Acad. Sci. U. S. A. 97:3948-3953.

13. Park, H.J., et al. 2002. Role of sterol regulatory element binding proteins in the regulation of Galpha(i2) expression in cultured atrial cells. Circ. Res. 91:32-37.

14. Buxton, A.E., et al. 2000. Electrophysiologic testing to identify patients with coronary artery disease who are at risk for sudden death. Multicenter Unsustained Tachycardia Trial Investigators. N. Engl. J. Med. 342:1937-1945.

15. Gomes, J.A., et al. 1997. Predictors of long-term survival in patients with malignant ventricular arrhythmias. Am. J. Cardiol. 79:1054-1060.

16. Brown, M.S., and Goldstein, J.L. 1980. Multivalent feedback regulation of HMG CoA reductase, a control mechanism coordinating isoprenoid synthesis and cell growth. J. Lipid Res. 21:505-517.

17. Shimano, H., et al. 1996. Overproduction of cholesterol and fatty acids causes massive liver enlargement in transgenic mice expressing truncated SREBP-1a. J. Clin. Invest. 98:1575-1584.

18. Shimano, H., et al. 1997. Elevated levels of SREBP-2 and cholesterol synthesis in livers of mice homozygous for a targeted disruption of the SREBP-1 gene. J. Clin. Invest. 100:2115-2124.

19. Corey, S., Krapivinsky, G., Krapivinsky, L., and Clapham, D.E. 1998. Number and stoichiometry of subunits in the native atrial G-protein-gated $\mathrm{K}^{+}$ channel, IKACh. J. Biol. Chem. 273:5271-5278.

20. Schwartz, P.J., La Rovere, M.T., and Vanoli, E. 1992. Autonomic nervous system and sudden cardiac death. Experimental basis and clinical observations for post-myocardial infarction risk stratification. Circulation. 85(1 Suppl.):I77-I91.

21. Antman, E.M., and Braunwald, E. 2001. Acute myocardial infarction. In Heart disease: a textbook of cardiovascular medicine. E. Braunwald, D.P. Zipes, and P. Libby, editors. W.B. Saunders. Boston, Massachusetts, USA. 1114-1218.

22. Virag, J.I., and Murry, C.E. 2003. Myofibroblast and endothelial cell proliferation during murine myocardial infarct repair. Am. J. Pathol. 163:2433-2440.

23. Powell, D.W., et al. 1999. Myofibroblasts. I. Paracrine cells important in health and disease. Am. J. Physiol. 277:C1-C9.

24. Donahue, J.K., et al. 2000. Focal modification of electrical conduction in the heart by viral gene transfer. Nat. Med. 6:1395-1398.

25. Liang, B.T., Hellmich, M.R., Neer, E.J., and Galper, J.B. 1986. Development of muscarinic cholinergic inhibition of adenylate cyclase in embryonic chick heart. Its relationship to changes in the inhibitory guanine nucleotide regulatory protein. J. Biol. Chem. 261:9011-9021.

26. Ward, S.M., Desgrosellier, J.S., Zhuang, X., Barnett, J.V., and Galper, J.B. 2002. Transforming growth factor beta (TGFbeta) signaling via differential activation of activin receptor-like kinases 2 and 5 during cardiac development. Role in regulating parasympathetic responsiveness. J. Biol. Chem. 277:50183-50189.

27. Ward, S.M., et al. 2002. TGFbeta regulates the expression of $\mathrm{G}$ alpha(i2) via an effect on the localization of ras. J. Mol. Cell. Cardiol. 34:1217-1226.

28. Vanoli, E., et al. 1991. Vagal stimulation and prevention of sudden death in conscious dogs with a healed myocardial infarction. Circ. Res. 68:1471-1481.

29. Kataoka, M., Ito, C., Sasaki, H., Yamane, K., and Kohno, N. 2004. Low heart rate variability is a risk factor for sudden cardiac death in type 2 diabetes. Diabetes Res. Clin. Pract. 64:51-58.

30. Burger, A.J., Charlamb, M., and Sherman, H.B. 1999. Circadian patterns of heart rate variability in normals, chronic stable angina and diabetes mellitus. Int. J. Cardiol. 71:41-48.

31. Aronson, D. 1997. Pharmacologic modulation of autonomic tone: implications for the diabetic patient. Diabetologia. 40:476-481.

32. O'Brien, I.A., McFadden, J.P., and Corrall, R.J. 1991. The influence of autonomic neuropathy on mortality in insulin-dependent diabetes. Q. J. Med. 79:495-502.

33. Callans, D.J., and Josephson, M.E. 2004. Ventricular tachycardias in electrophysiology from cell to bedside. D.P. Zipes and J. Jalife, editors. W.B. Saunders. Philadelphia, Pennsylvania, USA. 569-574.

34. Maguire, C.T., et al. 2003. Implications of ventricular arrhythmia vulnerability during murine electrophysiology studies. Physiol. Genomics. 15:84-91.

35. Vyas, A.K., et al. 2006. Reduction in ventricular tachyarrhythmias with statins in the Multicenter Automatic Defibrillator Implantation Trial (MADIT)-II. J. Am. Coll. Cardiol. 47:769-773.

36. Goldberger, J.J., et al. 2006. Effects of statin therapy on arrhythmic events and survival in patients with nonischemic dilated cardiomyopathy. J. Am. Coll. Cardiol. 48:1228-1233.

37. De Sutter, J., Tavernier, R., De Buyzere, M., Jordaens, L., and De Backer, G. 2000. Lipid lowering drugs and recurrences of life-threatening ventricular arrhythmias in high-risk patients. J. Am. Coll. Cardiol. 36:766-772.

38. Gadbut, A.P., et al. 1997. Induction of the cholesterol metabolic pathway regulates the farnesylation of RAS in embryonic chick heart cells: a new role for ras in regulating the expression of muscarinic receptors and G proteins. EMBO J. 16:7250-7260.

39. Schoots, O., Voskoglou, T., and Van Tol, H.H. 1997. Genomic organization and promoter analysis of the human G-protein-coupled $\mathrm{K}+$ channel Kir3.1 (KCNJ3/HGIRK1). Genomics. 39:279-288.

40. He, T.C., et al. 1998. A simplified system for generating recombinant adenoviruses. Proc. Natl. Acad. Sci. U.S. A. 95:2509-2514.

41. Kim, J.B., et al. 1998. Nutritional and insulin regulation of fatty acid synthetase and leptin gene expression through ADD1/SREBP1.J. Clin. Invest. 101:1-9.

42. Vallett, S.M., Sanchez, H.B., Rosenfeld, J.M., and Osborne, T.F. 1996. A direct role for sterol regulatory element binding protein in activation of 3-hydroxy3-methylglutaryl coenzyme A reductase gene. J. Biol. Chem. 271:12247-12253.

43. Patten, R.D., et al. 1998. Ventricular remodeling in a mouse model of myocardial infarction. Am. J. Physiol. 274:H1812-H1820. 